Wimmer, Michael

\title{
Antihumanismus, Transhumanismus, Posthumanismus: Bildung nach ihrem Ende
}

Kluge, Sven [Hrsg.]; Steffens, Gerd [Hrsg.]; Lohmann, Ingrid [Hrsg.]: Menschenverbesserung Transhumanismus. Frankfurt am Main [u.a.] : Lang 2014, S. 237-265. - (Jahrbuch für Pädagogik; 2014)

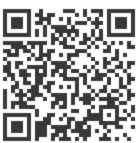

Quellenangabe/ Reference:

Wimmer, Michael: Antihumanismus, Transhumanismus, Posthumanismus: Bildung nach ihrem Ende - In: Kluge, Sven [Hrsg.]; Steffens, Gerd [Hrsg.]; Lohmann, Ingrid [Hrsg.]: Menschenverbesserung Transhumanismus. Frankfurt am Main [u.a.] : Lang 2014, S. 237-265 - URN:

urn:nbn:de:0111-pedocs-128248 - DOI: 10.25656/01:12824

https://nbn-resolving.org/urn:nbn:de:0111-pedocs-128248

https://doi.org/10.25656/01:12824

in Kooperation mit / in cooperation with:

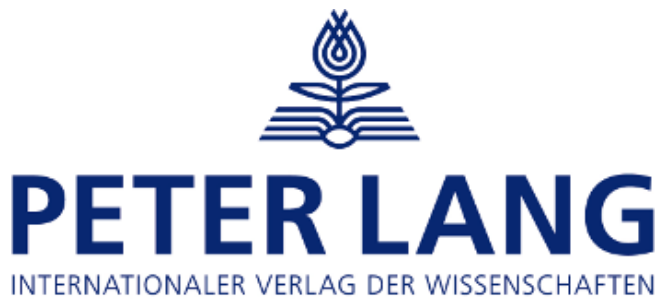

http://www.peterlang.com

\section{Nutzungsbedingungen}

Gewährt wird ein nicht exklusives, nicht übertragbares, persönliches und beschränktes Recht auf Nutzung dieses Dokuments. Dieses Dokument ist ausschließlich für den persönlichen, nicht-kommerziellen Gebrauch bestimmt. Die Nutzung stellt keine Übertragung des Eigentumsrechts an diesem Dokument dar und gilt vorbehaltlich der folgenden Einschränkungen: Auf sämtlichen Kopien dieses Dokuments müssen alle Urheberrechtshinweise und sonstigen Hinweise auf gesetzlichen Schutz beibehalten werden. Sie dürfen dieses Dokument nicht in irgendeiner Weise abändern, noch dürfen Sie dieses Dokument für öffentliche oder kommerzielle Zwecke vervielfältigen, öffentlich ausstellen, aufführen, vertreiben oder anderweitig nutzen.

Mit der Verwendung dieses Dokuments erkennen Sie die Nutzungsbedingungen an.

\section{Terms of use}

We grant a non-exclusive, non-transferable, individual and limited right to using this document.

This document is solely intended for your personal, non-commercial use. Use of this document does not include any transfer of property rights and it is conditional to the following limitations: All of the copies of this documents must retain all copyright information and other information regarding legal protection. You are not allowed to alter this document in any way, to copy it for public or commercial purposes, to exhibit the document in public, to perform, distribute or otherwise use the document in public.

By using this particular document, you accept the above-stated conditions of use.

\section{Kontakt / Contact:}

peDOCS

DIPF | Leibniz-Institut für Bildungsforschung und Bildungsinformation Informationszentrum (IZ) Bildung

E-Mail: pedocs@dipf.de

Internet: www.pedocs.de

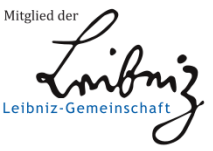




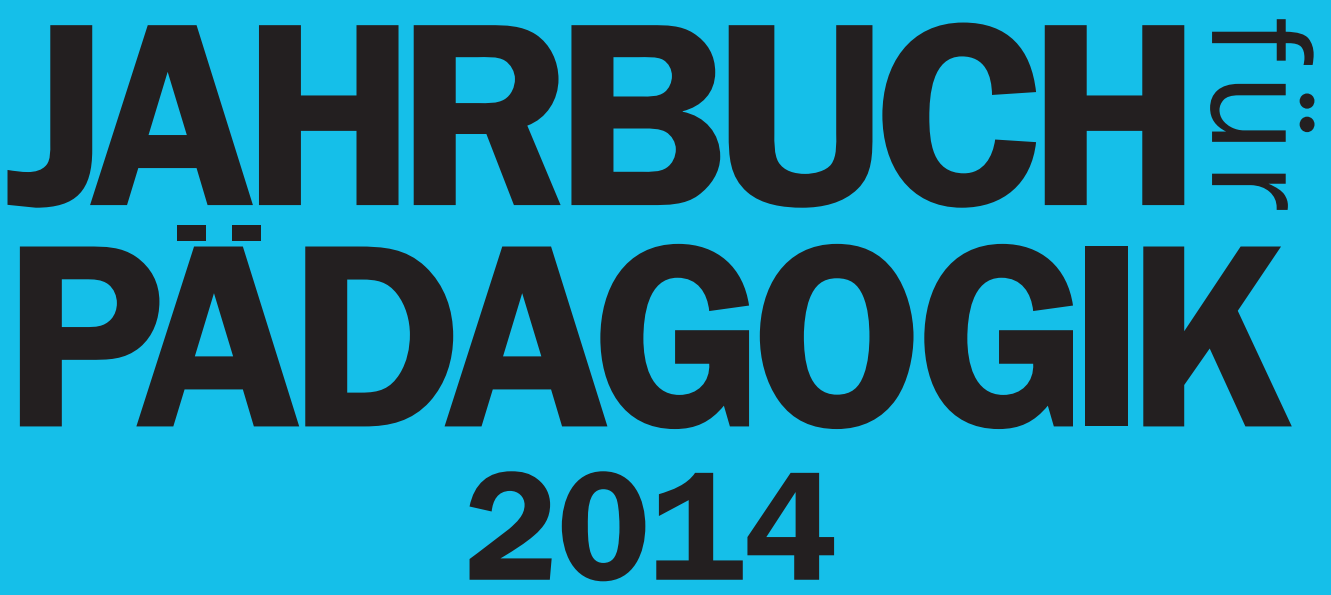

\section{Menschenverbesserung \\ Transhumanismus}


Die Debatte über Transhumanismus als kommender Zustand der Menschheit jenseits des homo sapiens ist mittlerweile aus dem Bereich futuristischer Phantasien in den von Diskursen übergegangen, in denen sich Projekte und Leitbilder der Zukunftsgestaltung formen. Es liegt auf der Hand, dass eine solche - als möglich und wahrscheinlich debattierte - Umwälzung der condition humaine eine Pädagogik beunruhigen und interessieren muss, die in Konzepten von Mündigkeit und Autonomie wie auch in Fragen des Generationenverhältnisses den Ankerpunkt ihrer Selbstverständnisse sieht. Das Jahrbuch für Pädagogik 2014: Menschenverbesserung - Transhumanismus diskutiert die dadurch aufgeworfenen Fragen in drei thematischen Schwerpunkten: I. Menschenverbesserung in der Moderne; II. Transhumanismus - Prognose oder Projekt? Ansätze, Diskurse, Entwicklungen; III. Jenseits des Menschen? Konsequenzen und Effekte für Bildung und (Selbst-) Erziehung.

Sven Kluge, Dr. phil., ist Lehrbeauftragter an der Universität Duisburg-Essen und seit 2009 Mitglied des Herausgeberkreises des Jahrbuchs für Pädagogik. Ingrid Lohmann, Dr. phil., ist Professorin für Ideen- und Sozialgeschichte der Erziehung/Historische Bildungsforschung an der Universität Hamburg und untersucht Beziehungen zwischen pädagogischem und ökonomischem Diskurs in der Neuzeit.

Gerd Steffens, Prof. Dr. phil., lehrte bis 2007 Politische Bildung und ihre Didaktik am Fachbereich Gesellschaftswissenschaften der Universität Kassel. Gegenwärtig forscht er zum Thema Transformationskrisen und gesellschaftliche Lernprozesse und ist Mitglied im Wissenschaftlichen Beirat einer NRO. 
Jahrbuch für Pädagogik 2014

Menschenverbesserung

Transhumanismus 


\title{
JAHRBUCH FÜR PÄDAGOGIK 2014
}

\author{
Menschenverbesserung \\ Transhumanismus
}

Redaktion:

Sven Kluge - Ingrid Lohmann - Gerd Steffens 


\section{JAHRBUCH FÜR PÄDAGOGIK}

Begründet von:

Kurt Beutler, Ulla Bracht, Hans-Jochen Gamm, Klaus Himmelstein, Wolfgang Keim, Gernot Koneffke, Karl-Christoph Lingelbach, Gerd Radde, Ulrich Wiegmann, Hasko Zimmer

HerausgeberInnen:

Martin Dust, Hannover

Sven Kluge, Münster

Andrea Liesner, Hamburg Ingrid Lohmann, Hamburg

David Salomon, Siegen

Jürgen-Matthias Springer, Essen

Gerd Steffens, Kassel

Edgar Weiß, Siegen 
Bibliografische Information der Deutschen Nationalbibliothek Die Deutsche Nationalbibliothek verzeichnet diese Publikation in der Deutschen Nationalbibliografie; detaillierte bibliografische Daten sind im Internet über http://dnb.d-nb.de abrufbar.

ISSN 0941-1461

ISBN978-3-631-65764-5 (Print)

E-ISBN 978-3-653-05104-9(E-Book)

DOI 10.3726/978-3-653-05104-9

(C) Peter Lang GmbH Internationaler Verlag der Wissenschaften Frankfurt am Main 2014 Alle Rechte vorbehalten.

Peter Lang Edition ist ein Imprint der Peter Lang GmbH. Peter Lang - Frankfurt am Main • Bern • Bruxelles • New York $\bullet$ Oxford $\bullet$ Warszawa $\bullet$ Wien

Das Werk einschließlich aller seiner Teile ist urheberrechtlich geschützt. Jede Verwertung außerhalb der engen Grenzen des Urheberrechtsgesetzes ist ohne Zustimmung des Verlages unzulässig und strafbar. Das gilt insbesondere für Vervielfältigungen, Übersetzungen, Mikroverfilmungen und die Einspeicherung und Verarbeitung in elektronischen Systemen.

Diese Publikation wurde begutachtet.

www.peterlang.com 


\section{Antihumanismus, Transhumanismus, Posthumanismus: Bildung nach ihrem Ende}

Zusammenfassung: Die Diskurse um das Ende des Menschen und der Topos vom Ende humanistischer Bildung erfahren durch den Transhumanismus nicht nur eine ungeahnte Reaktualisierung, sondern durch die Verschiebung der Referenz der Aussagen von der metaphorischen Ebene auf die des buchstäblich Realen zugleich eine Radikalisierung von größter Ambivalenz. Die in Aussicht gestellte Erfüllung ältester Menschheitsträume geht einher mit der Befürchtung des Schlimmsten. Man verspricht die Überwindung der Endlichkeit des Menschen und seine Vervollkommnung durch Supplementierung der Kulturpraktiken von Erziehung und Bildung durch Naturwissenschaft und Technik. Damit stellt der Transhumanismus für die Pädagogik eine fundamentale Herausforderung dar, auf die sie jedoch schlecht vorbereitet ist. Für eine radikale Kritik am Projekt der Verschmelzung von Mensch und Maschine, die nicht in den alten Dualismus von Vitalismus vs. Mechanismus und den Gegensatz von Bildung und Technik zurückfällt, fehlen ihr die theoretischen Mittel. Der Beitrag versucht daher, die diskursiven Ausgangskonstellationen zu skizzieren, von denen aus eine kritisch-theoretische Analyse des techno-theologischen Phantasmas des Transhumanismus möglich werden könnte.

Abstract: With the emergence of transhumanism the discourse about the end of man and the topos of the end of humanistic education and Bildung not only did undergo an unsuspected reactualization, but also a highly ambivalent radicalization, with the reference of statements shifting from a metaphorical to a literally real level. The prospect of fulfilling some of mankind's oldest dreams is accompanied by a fear of the worst. The supplementation of the cultural practices of education with the help of science and technology promises to overcome the finitude of man and bring him closer to perfection. Thus, transhumanism represents a fundamental challenge for pedagogy - to which it is ill-prepared. Pedagogy lacks the theoretical resources for a radical critique of the project of merging man and machine that does not fall back to dualistic positions like vitalism vs. mechanism or Bildung vs. technology. This article attempts to outline the discursive constellations from which a critical-theoretical analysis of the techno-theological phantasm of transhumanism might be possible.

Keywords: Posthumanismus, Anthropotechnologie, Selbstoptimierung, Bildung 


\section{Vorbemerkung}

„Die dringlichste Bildungsaufgabe besteht darin, das Bewußtsein des Menschen von sich selber auf die Höhe der technologischen Revolution zu bringen. Bildung hat es mit dem Bewußtsein des Menschen zu tun; das Hinstarren auf seine materielle Bedingung allein hebt sie nicht auf, obwohl die Erkenntnis dieser Bedingung unverzichtbar ist. Aufgabe der Bildung ist das Studium des Bewußtseins. [...] Die Herstellung der Handlungsfähigkeit gegenüber der technologischen Revolution ist das vornehmste Problem der Bildung [...] Befreiung des Bewußtseins durch Bildung stellt somit die Frage, wie der menschenzerstörende Bewußtseinsrückstand gegenüber der materiellen Produktion aufgehoben werden kann. [...] Mit der permanenten technologischen Revolution zeugt die Bildung die permanente Revolution des Menschen. [...] Der Mensch kann Mensch werden, sein eigener Täter, in seine eigene, menschliche Welt entlassen werden. Dies ist die letzte Forderung des Humanismus, sein Heraustreten aus der Welt der entfremdeten Dimension und des in ihr aufbewahrten Bedürfnisses und sein Eintreten in die Wirklichkeit. Es ist dies seine wahre Realisierung, seine letzte und höchste Würde, mit der seine gesamte Vergangenheit gerechtfertigt wird oder dem endgültigen Verdikt verfällt." (Heydorn 1972, S. 122ff, Herv. MW).

Diese Sätze schrieb vor über 40 Jahren Heinz-Joachim Heydorn in seinen Ausführungen zu einer Neufassung des Bildungsbegriffs. Sie enthalten alles, worum es auch hier gehen wird, sie formulieren allerdings das, was mir ein dringliches und keineswegs bereits geklärtes Problem zu sein scheint, in Form von solchen Antworten, die selbst zum Problem gehören: erstens die dichotomisierende Unterscheidung zwischen Humanismus, Bewusstsein, Bildung auf der einen und Technik, Entfremdung, Materialität auf der anderen Seite, die selbst zur kritisierten Herrschaftslogik gehört, zweitens die Ermächtigung des Menschen über seine materiellen Bedingungen zwecks Selbstüberschreitung hin zur Selbstschöpfung (,sein eigener Täter“ werden), drittens die mit dem Entfremdungsbegriff verbundene Figur der Wiederaneignung und Vereinnahmung des Fremden, nach der es darum geht, das dem Menschen fremd Erscheinende als etwas ihm Eigenes zu durchschauen, anstatt das vermeintlich Eigene als etwas Fremdes zu erkennen (Pfaller 2008, S. 226ff), und viertens die eschatologisch-apokalyptische Konzeption von Geschichte, die sich entweder als eine Vollendung des Humanismus in Form einer Offenbarung der Wahrheit des Menschen erfüllen wird, die alle Gewalt der Geschichte nachträglich legitimiert und mit Sinn erfüllt, oder die jedes Sinns beraubt in einem Nihilismus untergehen wird.

Diese Auffassung Heydorns von der Aufgabe und dem Ziel von Bildung im Spätkapitalismus ist m.E. aus heutiger Perspektive nicht nur überholt, sondern selbst fragwürdig. Seine Problematisierung der technologischen Revolution und 
der Bedeutung der technischen Bedingungen des Menschen und seines Selbstverständnisses jedoch ist zugleich höchst aktuell, denn die Fragen nach der Bildung und dem Menschen halte ich angesichts der gesellschaftlichen und politischen, aber maßgeblich auch wegen der rasanten wissenschaftlich-technischen Entwicklungen für dringlich, da es sich um Entwicklungen handelt, die nicht nur die Existenz des Menschen in all seinen Dimensionen betreffen, sondern die auch das Potential bergen, dass sich eine neue, global vernetzte und agierende Elite der neuen Technologien bemächtigt, die den Durchgriff auf die Grundelemente der Materie, des organischen Lebens, der neuronalen Prozesse und der Informationsund Kommunikationssysteme erlauben und damit ihre Steuerung, Rekombinierung und Neugestaltung. Zugleich stellen diese Entwicklungen, die das kulturelle Selbstverständnis der Menschen in seinen Grundlagen in Frage stellen, für den bildungstheoretischen Diskurs eine kaum zu ignorierende, aber auch kaum zu bewältigende Herausforderung dar, da sie im pädagogischen und insbesondere im bildungstheoretischen Diskurs ein entscheidendes systematisches Defizit offenbar werden lassen. Dieses besteht, radikal und etwas riskant formuliert, in einem gewissen Nicht-Denken des Technischen, das als etwas Äußerliches, Sekundäres, rein Instrumentelles und Objekthaftes aufgefasst und dem Menschen als Subjekt entgegengesetzt wird. Das Verhältnis zwischen Bildung und Technik wie allgemein zwischen Geistes- und Naturwissenschaften lässt sich auf diese Weise kaum angemessen erfassen, so dass auch eine von derartigen Unterscheidungen ausgehende Kritik an den aktuellen Entwicklungen, wie sie in Zusammenhang mit dem Transhumanismus zu beobachten sind, ohnmächtig bleiben muss und eher auf eigene Schwächen verweist. Statt die gegenwärtigen Entwicklungen aus einer bildungstheoretischen Perspektive angemessen zu erfassen und die mit ihnen gegebenen Möglichkeiten wie auch die Gefahren zu begreifen, geraten kritische Diskurse entweder leicht in den Sog eines technikfeindlichen Kulturpessimismus oder sie erliegen ebenfalls nur allzu leicht der Verführungskraft eines Technikfetischismus oder den Versprechungen bzw. der frohen Botschaft der Transhumanisten, die Menschheit von ihrer Unvollkommenheit, Endlichkeit und Sterblichkeit zu befreien.

Kurz: die Tradition - sei sie geisteswissenschaftlich, hermeneutisch, pragmatisch, sozialwissenschaftlich oder kritisch-rationalistisch geprägt - stellt uns keine Mittel zur Verfügung, die wissenschaftlich-technische Entwicklung in ihren Konsequenzen für die Zukunft von Mensch und Gesellschaft zu begreifen, ohne in die von der Denktradition vorgefertigte Falle vermeintlicher Alternativen zu geraten. Deshalb halte ich es für angebracht den Versuch zu machen, die Voraussetzungen noch einmal zu rekapitulieren, um die Situation etwas klarer bestimmen und das Problem deutlicher begreifen zu können. Deshalb möchte ich einige Fragen noch einmal aufnehmen (vgl. Wimmer 1988, S. i-iv), die weiterhin offen und vielleicht 
immer noch nicht richtig gestellt wurden, da sie vielleicht auch zu komplex und in sich heterogen sind, um in einem Anlauf bearbeitet und geklärt werden zu können, handelt es sich dabei doch um Probleme, die die kategorialen und epistemologischen Grundlagen unseres Welt- und Selbstverständnisses betreffen, wie z.B. das Verhältnis zwischen Mensch, Natur und Technik, zwischen Geist, Materie und Zeit, aber auch zwischen Medien, Wissen und Bildung oder die Beziehungen zwischen Identität, Alterität, Unbestimmtheit und Differenz.

Um eventuell zu hohe Erwartung von vornherein zu dämpfen sei vorangeschickt, dass ich nicht vorhabe die Frage, was „posthumanistische Bildung“ heißen könnte, zu beantworten, da ich noch nicht einmal weiß, ob eine Antwort darauf heute bereits möglich wäre. Es geht lediglich darum, das Terrain zu sondieren, um etwas mehr Klarheit darüber zu bekommen, wonach eigentlich zu fragen wäre, wenn man heute nach der Möglichkeit von Bildung fragt. Mit Blick auf die heutigen Möglichkeiten der Selbsterschaffung des Menschen, die alles in den Schatten stellen, was gegenwärtig vor allem in der Kulturwissenschaft (Villa 2008; Coenen u.a. 2010), der Körpersoziologie (Schroer 2005) und der Erziehungswissenschaft (Mayer u.a. 2013) unter der Optimierung des Selbst diskutiert wird, soll eine Problematik verdeutlicht werden, die nicht nur von bildungstheoretischer Relevanz, sondern auch gesellschaftlich, politisch und ethisch zukunftsentscheidend ist. Beginnen möchte ich (1) mit einer Erinnerung an die diversen Abschreibungen und Rettungsversuche von Bildung. Danach werde ich (2) kurz darauf eingehen, warum es so schwierig ist, etwas über die Zukunft von Bildung zu sagen und daran anschließend wird es dann um die These vom Ende des Menschen gehen (3) und um zwei konträre Interpretationen dieses Endes, den Transhumanismus (4) und den Posthumanismus (5). Abschließend (6) wird die Vermutung artikuliert, dass ein dekonstruktiver Perspektivwechsel eine Möglichkeit sein könnte, nicht nur das gebildete Bewusstsein, sondern auch die Bildungstheorie auf die von Heydorn geforderte „Höhe der technologischen Revolution zu bringen“.

\section{„Bildung“ zwischen Abschreibung, Verwirklichung und Transformation}

Im Unterschied zu Heydorn, der die damals aktuelle Aufgabe von Bildung mit großer Gewissheit glaubte bestimmen zu können, besteht im Gegenwartsdiskurs eine Spaltung zwischen denjenigen, die optimistisch die ökonomische Funktionalisierbarkeit von Bildung propagieren, und denjenigen, die gerade auch angesichts dieser Reduktion des neuhumanistischen Bildungsverständnisses eine fundamentale Unsicherheit selbst hinsichtlich der Möglichkeit von Bildung formulieren. Diese Diskurse reihen sich ein in die schon seit Jahrzehnten immer wiederkehrenden Reden von einem Ende der humanistischen Bildung oder der Bildungsidee 
Humboldtscher Provenienz, denen jeweils korrespondierende Gegenreden sowie Vorschläge antworten, den Bildungsbegriff durch andere, vermeintlich aktuellere oder konkretere Begriffe zu ersetzen oder den Bildungsbegriff neu zu fassen. Dazu einige knappe Stichworte.

Die Kritik am neuhumanistischen Bildungsgedanken ist so alt wie er selbst, doch erst mit Nietzsche erreichte diese Kritik eine Radikalität, die ihn als einen Wahn diagnostiziert, als eine pure Illusion, die allerdings durch ,jene(n) matte(n) Glanz, jenes rätselhafte Milchstrassen-Licht, welches um diese Bildung leuchtet", eine große Verführungskraft besessen habe (Nietzsche 1988b, S. 164). Hatte sich für Nietzsche Bildung schon reduziert auf ein blosses Wissen um die Bildung (Nietzsche 1988a), so konnte Adorno in seiner „Theorie der Halbbildung“ (Adorno [1959] 1997) nur noch einen Willen zum Schein von Bildung erkennen, der ihren Verlust nur notdürftig kaschierte, bevor Liessmann in seiner „Theorie der Unbildung" (2006) konstatierte, dass in unserer Zeit die Idee von Bildung selbst jede Legitimität verloren habe und damit selbst verschwunden sei, so dass ihr Fehlen noch nicht einmal mehr als Mangel verstanden werden könne. Bildung habe nicht nur aufgehört, „Ziel und Maßstab für die zentralen Momente der Wissensproduktion, der Wissensvermittlung und der Wissensaneignung zu sein“ (Liessmann 2006, S. 9f), vielmehr wäre das Verschwinden der Bildungsidee geradezu die Voraussetzung für den heutigen Umgang mit Wissen, das ,zu einer bilanzierbaren Kennzahl des Humankapitals" geworden sei. Unbildung ist in seinen Augen daher die „notwendige Konsequenz der Kapitalisierung des Geistes“. Mit einer stärker kulturkritischen Färbung und weniger auf Wissen und mehr auf Bilder, digitale Technologien und Kunst bezogen bekräftigt Maset diese Diagnose in seinem Essay „Geistessterben“ (2010). Dezenter und vorsichtiger, aber grundsätzlich ähnliche Befürchtungen artikulieren die vielen Stimmen, die sich kritisch mit den neuen bildungspolitischen Zielformulierungen und Reformen auseinandersetzen, die im Kontext des ökonomischen Neoliberalismus, der freien Marktideologie und der spätkapitalistischen Individualisierungsprozesse nicht nur alle Institutionen des Bildungssystems durchdrungen, sondern auch das Selbstverständnis der Adressaten wie auch der Professionen grundlegend verändert haben. (Schöller 2006; Pongratz 2009) Ein Ende der Bildung wurde befürchtet, wenn sie vollends zur Ware werde (Lohmann/Rilling 2002), wenn sie kompetenztheoretisch umgeschrieben und auf Bildungsstandards bezogen (Gruschka 2007), wenn sie auf Anpassungsleistungen im Sinne von Problemlösungskompetenzen und damit auf gesellschaftliche Brauchbarkeit (Ruhloff 1997; 2002) oder wenn sie auf Ausbildung und marktgängige Qualifikationen reduziert werde (Rößer 2006; Zymek 2005).

Abgesehen von den destruktiven Folgen, die diese Prozesse für die Individuen, aber vor allem für die Sozialität und Solidarität des Zusammenlebens haben 
würden (Masschelein/Simons 2002), wurde auch geradezu konträr dazu, also ihren Niedergang begrüßend, der Zusammenhang von Bildung und Macht erkannt, d.h. dass die Bildungsidee an ihrem Scheitern selbst beteiligt sei, da sich über sie die Individualisierung als spezifische Form machtförmiger Subjektivierung realisiert habe (Masschelein/Ricken 2003). Plädiert wurde dafür, den Bildungsbegriff völlig abzuschreiben. Auch aus einer anderen Perspektive betrachtet, der medientheoretischen Kittlers, artikulierte sich im Bildungsbegriff wie schon in der Phänomenologie des Geistes ohnehin immer nur die Weltinnenansicht eines durch die Buchkultur formierten Geistes, weshalb mit der „Austreibung des Geistes aus den Geisteswissenschaften" (Kittler 1980) zusammen mit DEM Menschen auch der humanistische Begriff von Bildung obsolet geworden wäre. Dieser Diagnose schloss sich auch Sloterdijk an, als er in seiner Elmauer Rede erklärte, dass die „Ära des neuzeitlichen Humanismus als Schul- und Bildungsmodell [...] abgelaufen [ist], weil die Illusion nicht länger sich halten läßt, politische und ökonomische Großstrukturen könnten nach dem amiablen Modell der literarischen Gesellschaft organisiert werden.“ (Sloterdijk 1999, S. 14) Und um einen letzten Zeugen aufzurufen, der die Frage nach der Bildung ebenfalls in der Spur Heideggers reflektiert, so konstatierte Nancy in aller Klarheit, falls man Heideggers Aussage vom Ende der Philosophie akzeptiere, sei „das Modell des in Bildung zu unterweisenden Subjekts nicht nur veraltet und überholt, sondern schlechthin nicht mehr philosophisch, das heißt, es bildet uns nicht mehr (als Individuen und Gesellschaft). Trotzdem deformiert es uns weiterhin“ (Nancy 1982, S. 235).

Gegenüber diesen Verlustanzeigen und Verfallsdiagnosen formierten sich jedoch auch schon früh Einsprüche. So wurde gegen die idealisierende Hochschätzung der allgemeinen Bildung eingewendet, dass die Wahrheit der Bildung gerade in der Berufsbildung läge, und gegen die Kritik an dem Verrat an der Humboldtschen Bildungsidee wendete z.B. Tenorth ein, dass es sich bei den Kompetenzmodellen und den Bildungsstandards ganz im Gegenteil um die Erfüllung seiner innersten Intentionen handelte (vgl. BMBF 2003, S. 66f). Auch dass Bildung ausschließlich an das Medium der Schrift bzw. des Buches gebunden sei, wurde als eine unhaltbare Behauptung zurückgewiesen (Meyer u.a. 2011). Da es Bildung nie ohne Medien gebe und gegeben habe, sei sie heute nur anders, aber nicht verschwunden. Aus einer ganz andern Perspektive auf den Bildungsdiskurs wurde wiederum deutlich gemacht, dass die Kriterien der Verteidiger wie der Kritiker des Bildungsbegriffs problematisch wären, sofern Idee und Realität, Anspruch und Wirklichkeit, Theorie und Empirie immer nur gegeneinander ausgespielt würden. Bei Bildung habe es sich aber von Anfang an immer nur um humanistische (Schäfer 1996) oder um „Notwenige Illusionen“ (Kubac 2007) gehandelt, zudem gehörten Bildung und Entbildung ebenso zusammen (Sattler 2009) wie Bildung es mit Widerfahrnissen und Widerständen zu tun habe (Thompson/Weiss 2008). 
Außerdem und grundsätzlich sei Bildung prinzipiell nur eine Möglichkeitskategorie, so dass es sich also spätestens seit der Moderne überhaupt immer nur um ein unmögliches Versprechen und eine Versprechen von etwas Unmöglichem gehandelt habe. (Schäfer 2011)

Da all diese Diskurse zumeist auch die Konsequenzen ihrer Kritik für das Verständnis von Bildung artikulieren, kann man sie ebenso als Teilnehmer an einem andauernden Prozess der Re- und Neuformulierung des Bildungsbegriffs lesen. Darüber hinaus lassen sich natürlich auch Versuche einer expliziten Neufassung nennen, wie z.B. denjenigen Heydorns (1972) oder Klafkis (2007), aber auch die Ansätze einer Theorie transformatorischer Bildungsprozesse (Koller 2012) oder die Versuche, Bildung anders (Pongratz 2010) oder ausgehend vom Anderen zu denken (Wimmer 1996). Wie auch immer die Vorschläge eines Neudenkens von Bildung begründet sind und zu bewerten sein mögen, stets geht es in der bildungstheoretischen Reflexion darum, einen Umgang mit den durch den Poststrukturalismus und die Systemtheorie ausgelösten Erschütterungen zu finden und eine theoretische Fassung für den Werdensprozess eines Subjekts zu suchen, das keines mehr ist, das nur als gespaltenes, als Leerstelle, als nicht mit sich identisches, sich selbst partiell fremdes und nie vollständig bei sich seiendes Wesen beschreibbar ist, das alle Attribute, die dem Menschen in seiner vermeintlichen Sonderstellung zukommen, nicht ohne andere und nicht ohne Techniken erlangen würde, allen voran Sprachwesen, Bewusstseinsträger und Vernunftinhaber zu sein. (Gamm 2004, S. 40ff; 63ff)

Hominiden werden „Menschen“ nur vermittels technischer, d.h. „unmenschlicher" Bedingungen. Dieser aus anthropozentrisch-humanistischer Perspektive skandalöse Satz könnte aus einer anderen, posthumanistischen Perspektive auch lauten, dass Mensch und Technik, Natur und Kultur immer schon eine Einheit bilden, ohne dasselbe zu sein. (Hardt/Negri 2002, S. 104ff) Doch wie lässt sich Subjektivität in Formen von Selbstbewusstsein und Gedächtnis zusammen denken mit ihren technischen Bedingungen, ohne dass es in abstrakter Umkehrung zu einem Technikzentrismus kommt? Wie entkommt man dem Gegensatz von Mensch - Maschine? Fragen wie diese entstehen zwangsläufig, wenn die kategorialen Grenzen und begrifflichen Unterscheidungen erodieren, ohne dass schon eine neue Konfiguration der Verhältnisse erkennbar geworden ist. Die Formulierung „Bildung nach ihrem Ende“ führt außerdem in die Irre, wenn sie als eine ausschließlich chronologische Abfolge verstanden wird, denn wie der kurze Abriss gezeigt hat, hat das Ende der Bildung den Bildungsdiskurs von Anfang an begleitet, und mehr noch, es war ein integraler Bestandteil des Bildungsbegriffs. Schon bei Schiller, Hegel und Humboldt war die Möglichkeit ihrer Verfehlung, ihrer Aufhebung, ihres Scheiterns und ihrer Unmöglichkeit immer schon mitgedacht. Das Ende der Bildung ist im Bildungsdiskurs so präsent wie das Versprechen 
(Schäfer 2011), gab es doch nie eine Garantie, sondern immer nur und im besten Fall die in Aussicht gestellte Möglichkeit. Das Ende der Bildung kommt also nicht nach einer Zeit, in der sie wirklich geworden wäre, sondern (neu)humanistische Bildung besteht von Beginn an nur im Aufschub ihres Endes im Sinne der Erkenntnis ihrer Unmöglichkeit. Auch heute verdient ja nicht jede Entwicklung oder Veränderung, Bildung genannt zu werden. „Bildung nach ihrem Ende“ kann dann nur heißen, die Unmöglichkeit dieser versprochenen Möglichkeit zu begreifen. Inmitten einer vollkommen kontingent gewordenen Welt, in der es kaum noch etwas Notwendiges gibt und alles auch anders möglich wäre, stellt sich dann die Frage, welche Möglichkeiten es noch gäbe, wenn alles nur noch möglich wäre und in der Virtualität jeden Bezug zum Realen verloren hätte. Könnte es dann noch etwas geben, womit man eine Erfahrung machen könnte, etwas Fremdes, unerwartet anderes, das eben nicht auch anders sein könnte als ,anders als Sein“ (Levinas 1992)? Bildung wäre dann wohl nur noch möglich, wenn es eine andere Möglichkeit des Möglichen geben könnte.

\section{Gespaltene Zukunft}

Wie also der Mensch und seine Bildung neu zu denken sind, nachdem sie bereits mehrfach überholt wurden und untergegangen sind, und ob es überhaupt möglich ist, ihre zukünftige Gestalt zu erfassen, ist keineswegs schon ausgemacht. Lars Gustafsson schrieb einmal: „Nicht die Grenze zwischen Wirklichkeit und Fiktion, sondern das paradoxe Verhältnis zwischen dem Anspruch, eine Vorhersage zu enthalten, und dem Anspruch, etwas Unvorhersagbares vorherzusagen, macht die Utopie zur Utopie.“ (Gustafsson 1985, S. 92) Diese Paradoxie der Utopie ließe sich vielleicht unsichtbar machen, wenn es gelingen könnte, das Utopische in Metaphern der Wirklichkeit zu übersetzen, was ich, selbst wenn es gelingen sollte, nicht vorhabe. Denn worum es geht, und zwar nicht nur heute und auch nicht allein mit Blick auf Bildung und Politik, ist die Schwierigkeit, etwas über die Zukunft zu sagen, was über die magere Auskunft hinausgehen könnte, dass alles auch anders möglich wäre und werden kann.

Seit Zukunft nicht mehr in den Kontext einer geschlossenen geschichtsphilosophischen Konzeption eingespert ist und auch nicht mehr in den theoretisch unbegründbaren Totalitätsentwürfen von Utopien imaginiert werden kann, sondern im Evolutionsprozess des Sozialen zur Chiffre und zum Platzhalter von Kontingenz herabgestimmt wurde, kann man über sie kaum noch etwas sagen. Das gilt insbesondere von der sich selbst so nennenden Wissensgesellschaft, in der man merkwürdigerweise so wenig von der Zukunft wissen kann, wie nie zuvor. Schließlich weiß man von künftigem Wissen noch nichts, da es dann ja bereits 
bekannt und also nicht mehr zukünftig wäre, und folglich wird die Gesellschaft immer unvorhersehbarer, je mehr sie von Wissen abhängig ist. Je wichtiger das Wissen für die Zukunft wird, desto weniger kann man etwas von der Zukunft wissen. Und so ist es kein Wunder, wenn in gegenwärtigen Zeitdiagnosen der gestiegenen Ungewissheit und der zunehmenden Bedeutung des Nichtwissens immer mehr Aufmerksamkeit gewidmet wird. (Böschen u.a. 2004; Wehling 2006)

Hatte die Theorie des Posthistoire ein End-Bild der westlichen Zivilisation gezeichnet, in der sich trotz unaufhörlicher Bewegungen und sich beschleunigender Prozesse im Grunde nichts wesentliches mehr ereignet, so dass es zu einem Stillstand der Geschichte kommt (Kamper 1986, S. 59ff) ${ }^{1}$ und die Zukunft in der Gegenwart verschwindet (Nowotny 1993, S. 9), so setzt die Wissensgesellschaft auf Evolution, indem sie auf Komplexität reagiert, statt Prognosen zu erstellen, und damit den Begriff von Zukunft selbst verschiebt. Wenn nach Luhmann der Evolutionsbegriff selbst Prognosen ausschließt (Luhmann 1998, S. 296), weil wir uns als Beobachter zum einen selbst bewegen und wir uns außerdem auf ein Ziel zu bewegen, das sich ebenfalls selbst bewegt, dann kann man die Zukunft nicht mehr voraussagen, sondern nur noch provozieren, und sei es durch Voraussagen. Was dabei herauskommt, kann man aber nicht wissen. Wenn es also vormodern immer anders kam, als gedacht, und man in der Moderne dagegen glaubte, es so kommen lassen zu können, wie man wollte, weiß man heute, dass es auch postmodern immer anders kommen kann als geplant, dass die Zukunft also offen ist und Handeln stets riskant. Zwar ist auch postmodern weder alles möglich noch nichts unmöglich, doch alles scheint auch anders möglich zu sein und sogar gewesen zu sein. Alle Zeithorizonte, seien sie prophetisch, eschatologisch, utopisch, geschichtsphilosophisch oder wie auch immer mythisch, religiös, metaphysisch fundiert gewesen, sind von derartigen teleologischen Erzählungen befreit und konfrontieren uns daher mit einer ebenso unvorhersehbaren wie auch vorbildlosen Zukunft.

Was kann man also über das Kommende sagen, außer dass man hofft, es noch erleben zu können? Dass man für das Kommende bereit sein und dass man lernen muss, die Ungewissheit zu lieben, gerade weil man nicht wissen kann, was die Zukunft bringt, was auf uns zu kommt, und ob das, was kommt, gut ist oder vielleicht sogar das Schlimmste? Wenn es aber darum geht, mit der Ungewissheit leben zu lernen, sich auf das Unvorhersehbare einzustellen und das Unerwartbare zu erwarten, wodurch unterscheidet sich diese Haltung (die derjenigen philosophischen Haltung ähnelt, der es darum ging, den Tod zu akzeptieren) dann noch von dem Imperativ gegenwärtiger Bildungsvorstellungen, stets und überall sich den sich schnell wandelnden Qualifikationserwartungen und den neuen Marktbedingungen anpassen zu können und auf Ungewissheiten durch eine fortwährende geschmeidige Umstellungsbereitschaft reagieren zu können? Wo liegt die Grenze 
zwischen der (ethisch-politischen) Offenheit für den Anderen, die Zukunft und das nicht Antizipierbare auf der einen Seite und der (ökonomisch-funktionalen) Unterwerfung unter die Marktgesetze von Angebot und Nachfrage? Kurz und formalisiert gesagt: Wie lässt sich ein Verhältnis zum Anderen denken, zum anderen Menschen, zum Tier, zur Technik, zur Zukunft, in dem die Differenz weder neutralisiert noch hierarchisch codiert, aber auch nicht als eingeschlossene Differenz in einer Identität aufgehoben wird?

\section{Die Enden des Menschen und seiner humanistischen Bildung}

Die Schlachten zwischen Vertretern der Moderne und der Postmoderne, Humanisten und Antihumanisten sind geschlagen, die rhetorischen Wolken haben sich verzogen und die ehemaligen Kampfbegriffe umgibt schon fast eine Patina, die an eine heute kaum noch vorstellbare Intensität einer öffentlichen philosophischen Auseinandersetzung erinnert, in der es um das Schicksal der Gesellschaft und der westlichen Kultur, um unsere Lebensformen und unser Selbstverständnis ging. Einige der damals noch provokanten Kernthesen sind zwar inzwischen zu Formeln erstarrt und zum Allgemeingut geworden, wie z.B. die vom „Ende der großen Erzählungen“ (Lyotard) oder der „Ambivalenzen der Moderne“ und des Fortschritts (Baumann), doch das Ungedachte und daher Skandalöse, was an diesen und anderen Themen damals die Gemüter erhitzte, ist nahezu in Vergessenheit geraten. Die Erschütterung der Denksysteme und der Grundlagen des kulturellen Selbstverständnisses, die sich in diesen Diskursen artikulierte, wurde überschrieben durch andere Selbstbeschreibungen der Gesellschaft, die griffiger waren, Orientierung versprachen, Halt gaben und als Rahmen für positive Analysen und die Formulierung von Zukunftsaufgaben dienten. Zu nennen wären vor allem die Begriffe Globalisierung und Wissensgesellschaft. Was jedoch schon damals nur ansatzweise und nicht konsequent diskutiert wurde, ist die These vom Ende des Menschen.

Die Diskussion um Post- und Transhumanismus ist daher keineswegs neu. Ihre Originalität hatte sie schon vor Foucaults berühmten Sätzen aus dem Schlusskapitel der „Ordnung der Dinge“ von 1966 verloren, die, wenn auch auf neue Weise, die Grundgedanken Nietzsches ebenso wiederholten wie diejenigen Heideggers aus dem Humanismusbrief. Und, um daran wenigstens zu erinnern, auch Adorno hatte bereits 1962 den Anthropozentrismus als hoffnungslos überholt kritisiert (Adorno 2003, S. 24) und noch vor ihm prägte Günther Anders schon 1956 mit Blick auf die zweite (und 1980 dann auf die dritte) industrielle Revolution die Metapher von der „Antiquiertheit des Menschen“. Und geht man noch weiter zurück, trifft man auf Turing, der 1950 die Sonderstellung des Menschen als animal 
rationale mit Blick auf das Verhältnis „Computing Machinery and Intelligence“ vehement bezweifelte (Turing 1987). Dass die Radikalität und Undurchlässigkeit der anthropologischen Grenzen zum Tier wie auch zur Maschine nicht erst seit kurzer Zeit, sondern schon seit sehr langer Zeit bezweifelt werden, zeigt sich aber nicht nur hinsichtlich des vermeintlichen Alleinstellungsmerkmals der Rationalität, sondern auch der Körperlichkeit. Erinnert sei an La Mettrie, dessen Buch „L'Homme Machine“ eine ernorme Wirkungsgeschichte aufweisen kann, aber auch an Leibniz, der im 64. Absatz seiner Monadologie den „organischen Körper (Leib) eines Lebendigen eine Art von göttlicher Maschine oder natürlichem Automaten“" nannte (Leibniz 1979, S. 28). Maschinen der Natur unterscheiden sich für ihn allein dadurch von künstlichen, dass sie ,noch Maschinen in ihren kleinsten Teilen bin ins Unendliche" seien. Und erinnert sei natürlich noch einmal an Nietzsche, für den der Mensch das „,nicht festgestellte Tier“ ist, „das Tier, das versprechen darf". Auf eine radikale Weise und heutige Positionen der Transhumanisten vorwegnehmende Weise begriff auch Samuel Butler 1863 in seinem Essay „Darwin among the Machines“ das Ende des Menschen als Krone der Schöpfung. „Dessen These lautet: Durch die Entwicklung mechanischer Apparaturen sind wir dabei, eine uns überlegene Spezies von Maschinenwesen heranzuzüchten, die in absehbarer Zukunft die Herrschaft über die Erde antreten wird." (Welsch 2004, S. 53) Zur Zeit der Dampfmaschinen imaginierte Butler bereits intelligente Maschinen mit der Fähigkeit zur Selbststeuerung und war zugleich davon überzeugt, dass es nur eine Frage der Zeit wäre, „when the machines will hold the real supremacy over the world and its inhabitants" (Butler 1863, zit. nach Welsch).

Nichts Neues also? Ich denke doch. Über die Zauberlehrlingsfigur einer Verselbständigung der eigenen technischen Geschöpfe hinaus, die von Objekten zu den eigentlichen Subjekten werden und mit dem Schöpfer so die Rollen tauschen, aber auch über die schlichte Identifizierung von Mensch und Maschine oder von Mensch und Tier, also über den Kurzschluss oder die abstrakte Negation der dichotomen Subjekt-Objekt-Logik hinaus sind heute Verhältnisse sowohl denkbar als auch auf dem Weg technischer Realisierbarkeit, die die starren Grenzen überwinden, ohne sie völlig zu zerstören, so dass sich das Eine im Anderen zur Geltung bringt, ohne es zu ersetzen oder mit ihm identisch zu werden. Kurz, bei Aufrechterhaltung der Differenz werden beide Seiten zu dem, was man das ausgeschlossene Dritte nennen könnte (Bedorf u.a. 2010), also sowohl A wie auch Nicht-A. Hybride Wesen, Cyborgs, Maschinenmenschen und Menschenmaschinen, paradoxe Realisierungen und Mischwesen, Chimären, die sowohl das eine wie auch das andere sind und zugleich weder das eine noch das andere, sondern etwas neues eigener Art. (Haraway 1995; Eßlinger u.a. 2010) Allerdings macht es einen Unterschied um das Ganze aus, ob es gelingt, das anthropozentrische 
Selbstverständnis in diesem Sinne aufzulösen, dass also die Entgegensetzung zum Tier oder der Maschine überwunden und das Verhältnis zum äußeren Anderen wie zur eigenen Heteronomie bzw. zu dem, was bislang als das Inhumane aus dem Verständnis des Menschlichen ausgegrenzt wurde, anerkannt wird (als eingeschlossene Differenz oder Alterität, als heteronome Bedingtheit, Selbstfremdheit oder Nichtidentität), oder ob nicht das Verständnis des Menschlichen (l'humain/human), sondern der reale Mensch (l'homme/man), der homo natura im Sinne einer zweiten Schöpfung oder einer nun vom Menschen selbst gesteuerten Evolution technologisch substituiert und vervollkommnet wird (vgl. Schirrmacher 2001).

Doch die Diskussion über die Konsequenzen der neuen technischen Möglichkeiten steht eigentlich noch aus. Zwar gab es Ende der 90er Jahre einen heftigen Ausbruch im Diskurs um die Gentechnik, der die alten Topoi von der Verselbständigung der Technik und der drohenden Hegemonie der Maschine über den Menschen hinter sich ließ. Doch statt um die Sache zentrierte sich die Diskussion um den Autor und um Nebenschauplätze. Peter Sloterdijks „Regeln für den Menschenpark“ führte nicht zu einer ernsthaften Auseinandersetzung mit der gentechnischen Herausforderung und einer Reformulierung eines kulturellen Selbstverständnisses nach dem Ende des Humanismus, sondern um die Problematik der Gegenwartsdiagnose des Autors (Rebarbarisierung durch die neuen Medien) und seinen Therapievorschlag (Elitebildung), um die fragwürdige Nähe seiner Überlegungen zu gut tabuierten NS-Themen wie Züchtung, Rasse und Eugenik und um die Spannungen zwischen Postmoderne und Kritischer Theorie, die gut dokumentiert ist als Sloterdijk-Habermas-Kontroverse. Kaum kommentiert und wenig diskutiert wurden dagegen weder die nach wie vor virulente Frage nach den Chancen und Folgeproblemen der Gentechnik und die von Sloterdijk angesprochene Unumgänglichkeit und Unlösbarkeit der mit der Gentechnik zusammenhängenden Probleme (Sloterdijk 1999, S. 47), noch die ins Spiel gebrachte Möglichkeit, pädagogische „Menschenzähmung“ mittels Lektüre und Bildung durch eine andere, wirksamere Anthropotechnik zu ersetzen. Und bevor noch wenigstens in Umrissen hätte erkennbar werden können, was es für den Menschen heißen könnte, durch das Klonen von der Antiquiertheit seines Leibes befreit werden zu können, über die Keimzelle das Humanum berechnen, die eigene Evolution steuern und damit sein Wesen radikal in Frage stellen zu können, entstand eine ethische Antwort auf diese Frage, die nicht nur zur Vorsicht mahnte, sondern den religiösen Humanismus gegen die wissenschaftlich-technischen Möglichkeiten wieder in Stellung brachte. Das Prinzip Verantwortung von Hans Jonas rekurrierte auf das Mysterium des Humanen und plädierte dafür, gegen den Frevel der Gentechnik, die das Tabu über die menschliche Kreatürlichkeit antastet, das Heilige des Humanum zu restituieren 
und in der entzauberten Welt „freiwillig neue Tabus aufzurichten“ (Jonas 1987, S. 218; vgl. auch Altner 1998).

Ich will mich nicht zum Fürsprecher der Gentechnik machen, doch halte ich diese ethische Antwort auf die technologischen Herausforderungen für unzureichend. Wenn wir uns nicht mehr vorstellen können, was wir herstellen können, wie Anders sagte, dann kann die Antwort m.E. nicht in einem Denkverbot oder einer schlichten Arbeitsniederlegung bestehen, sondern nur darin, die Konvergenztechnologien von Atomen, Genen, Neuronen und Bits als die eigenen Fragen unserer Zeit in ihren Möglichkeiten und Ambivalenzen anzuerkennen, d.h. auch als Drohungen des Schlimmsten. Moral, Werte und humanistische Ethik helfen hier kaum weiter, da sie die erforderliche Streitkultur verhindern und in einer Situation prinzipieller Unsicherheit und Angst vermeintlich sicheren Halt anbieten. Statt größerer Klarheit gewinnt man so nur Denkverbote. Anstelle also zur Kenntnis zu nehmen, dass mit den Atomen, den Genen, den Neuronen und den Bits die Grundbausteine unserer Welt entdeckt wurden und nun der technischen Synthese auf allen Ebenen unter der Regie der Machteliten in Wissenschaft, Wirtschaft, Militär und Politik zur Verfügung stehen, neigt die Öffentlichkeit zur Moralisierung der Problematik, anstatt ihre politische Bearbeitung anzustreben oder einzufordern, die die irreführende Pseudoalternative zwischen profitabler Machbarkeit und ethischer Regulierung überwindet.

Das schon in den 70er Jahren verkündete „Ende des Menschen“ durch den theoretischen Antihumanismus, der das Ende einer spezifischen Denkstruktur und des mit ihm verbundenen metaphysischen Humanismus gemeint hatte, wird also seit einigen Jahren auf der Ebene des Realen faktisch herbeigeführt als Beendigung des Hominiden und lebendigen Humanen durch Umbau und Verschmelzung mit der Maschine, ohne aber noch in dem Kontext, dem Problembewusstsein und der Geistesgegenwart vom Ende der 90er Jahre wahrgenommen und problematisiert zu werden. Vielmehr könnte man von einer Spaltung sprechen zwischen der sozial- und kulturwissenschaftlichen Problematisierung auf der einen Seite, die sich um die Fragen der Biopolitik, des Körpers und einer Optimierung des Selbst zentriert, also um die weichen Anthopotechniken der Prothetik, Athletik, Diätetik und Kosmetik, und auf der anderen Seite einer utopisch-technizistischen Arbeit an einer Entgrenzung des Menschen, seiner Cyborgisierung und Entkörperlichung durch die harten Anthropotechnologien, die „,bis zu einer expliziten Merkmalsplanung vordringen“ (Sloterdijk 1999, S. 46), zu Neu-Züchtungen und entsprechenden Selektionsfolgen. Es versteht sich beinahe von selbst, dass die Experten mit dem anthropotechnischen Königswissen einen politischen Führungsanspruch artikulieren und selbst da, wo sie es nicht explizit tun, sich ihrer selbst generierten evolutionären Vorteile und ihres Anspruchs auf eine biosoziale Führungsposition aufgrund ihrer größeren Überlebensmacht sicher sind. Doch was ist von Ihren Visionen selbst zu halten? 


\section{Transhumanismus}

Wie der Diskurs um die „Enden des Menschen“ (Derrida 1988) ist auch der Diskurs um den Posthumanismus sehr heterogen und umfasst ein großes Spektrum (Herbrechter 2009), das von einer technophilen transhumanistischen Vision einer realen Überwindung des Menschen durch seine Verschmelzung mit der Maschine über die technikkritische Ablehnung aller Eingriffe in die menschliche Natur, die von der in der Tradition des Humanismus stehenden Kritischen Theorie vertreten wird (Habermas 2001), bis hin zu einem dekonstruktiven Ansatz reicht, der gegen den metaphysisch-anthropozentrischen Humanismus und seine geschichtswirksamen Verkennungen und Destruktivkräfte gerichtet ist und für ein anderes (Selbst-) Verständnis des Menschlichen eintritt, für einen Posthumanismus im Sinne eines „Humanismus des anderen Menschen“ (Levinas 1989) z.B., nicht aber für seine reale Überwindung. Orientiert man sich an den beiden Extremen dieses Diskursfeldes, dem Trans- und dem Posthumanismus, so lässt sich diese Spaltung bereits bis Nietzsche zurückverfolgen, der einerseits in seiner radikalen Vernunft- und Subjektkritik mit der humanistischen Moral, dem rationalistischen Selbstverständnis der Aufklärung und der abendländischen Kultur bricht, um eine Neuinterpretation von Mensch und Welt nach dem Tod Gottes zu ermöglichen, der aber andererseits auch die praktische Überwindung des Menschen nicht nur gefordert, sondern erwartet hat, und als Prophet des Übermenschen aufgetreten ist.

Nietzsches Übermensch scheint nun in greifbare Nähe gerückt zu sein. Dank der technologischen Entwicklungen halten nicht wenige den Traum einer Selbstüberwindung nicht länger für einen Traum. Was angesichts des technologischen Wandels und des Siegesfeldzugs der Kybernetik auf dem Spiel zu stehen scheint, ist die Frage der menschlichen Natur (Hagner/Hörl 2008). Kaum ein Aspekt des Alltags ist von diesem Wandel nicht betroffen (Herbrechter 2009, S. 26ff), hat doch z.B. das „,technische System“ (Stiegler 2011) bereits einen neuen virtuellen Kontinent geschaffen, und die neuen techno-kulturellen Praktiken haben neue Subjektivitäten hervorgebracht. Doch nicht nur das Selbstverständnis und die Selbsttechnologien haben sich gewandelt, betroffen von den neuen Technologien ist das gesamte humanistische Kategorisierungs- und Ausgrenzungssystem. Zugleich scheinen uralte Träume, die Unvollkommenheiten und Mängel des Menschen überwinden zu können, in greifbare Nähe der Realisierbarkeit gerückt zu sein. So kann man in dem Film „Welt ohne Menschen“2 die technische Realisierung des bisher Unmöglichen beobachten: Lahme können wieder gehen (Wunder), der Geist herrscht über die Materie (Magie), er kann reale Prozesse steuern ohne einen Finger zu krümmen (Teleportie), man kann Gedanken lesen und das Gehirn direkt an den Rechner anschließen (Engel), man kann jemanden anrufen 
ohne noch wählen zu müssen (Telepathie), man kann den Geist real vom Körper trennen, ihn durch einen vollständigen Gehirnscan extrahieren und den Körper durch eine Maschine ersetzen, so dass das Bewusstsein potentiell unsterblich wird etc. Gewiss, es sind noch Phantasien, doch wie die Politik der Transhumanisten zeigt, keineswegs wirkungslos. So sagt ein früherer Student der Singularity University, David Dalrymple, im Film: „Wenn wir das Individuum von seinem fehlbaren, biologischen Körper abkoppeln können, dann müssen wir den Tod nicht mehr auf die selbe Weise betrachten wie heute. Indem wir ein Backup von uns selbst auf Harddisc erstellen, werden wir eine unbegrenzte Lebensdauer erreichen." Und Ray Kurzweil, einer der Mitbegründer der Singularity University und Meinungsführer: „In etwa 20 Jahren - ich habe das Datum 2029 gewählt - wird eine Maschine, eine künstliche Intelligenz, menschlicher Intelligenz ebenbürtig sein und sie sogar übertreffen. Unsere Gehirne werden zum größten Teil nicht biologisch und wir also im Grunde Maschinen sein. Wir können aufhören zu altern. Wir können ewig leben.“

Dietmar Kamper hat schon vor Jahren die Beobachtung gemacht, dass das Projekt der Moderne von Anfang an mit einem anderen Programm konkurrierte, „zur gegebenen Welt eine eigene Welt der Menschen mittels und nach Maßgabe der Einbildungskraft zu erschaffen“ (Kamper 1995, S. 7). Während die Aufklärung in ,postmoderne Nöte“ geraten sei, triumphiere dieses Programm, das alles Existierende als Bild deute, in Zeichen verwandele und über Datenverarbeitung auf Bildschirmen zugänglich mache. Diese maschinelle Immaterialisierung des Realen ,mag an der Verknüpfung des Imaginären mit dem uralten Traum von der Unsterblichkeit liegen, der einen Bogen von der ältesten Theologie zur neuesten Technologie zu spannen erlaubt.“ (Ebd.) Im Programm der Transhumanisten findet man diese religiös gespeiste Triebkraft der auf die Spitze getriebenen Selbstvervollkommnung und Weltbemächtigung bestätigt. Dabei spielt das Imaginäre und das Zum-Bild-Machen eine nicht unbedeutende Rolle, wie schon Heidegger in seinem Text „Zeit des Weltbildes“ ([1938] 1980) verdeutlichen konnte. Erst die Neuzeit begann, die Welt zum Bild zu machen und sich ihrer dadurch zu bemächtigen. Weltbild ,meint daher nicht ein Bild von der Welt, sondern die Welt als Bild [...] Das Seiende im Ganzen wird jetzt so genommen, daß es erst und nur seiend ist, sofern es durch den vorstellend-herstellenden Menschen gestellt ist." (Heidegger 1980, S. 86) „Wo die Welt zum Bild wird, kommt das System, und zwar nicht nur im Denken, zur Herrschaft.“ (Ebd., S. 98)

So betrachtet ist es nicht erstaunlich, dass man die Geburtsstunde des kalifornischen Denkens ziemlich genau datieren kann, denn nirgendwo hinterließ das 1968 von der Apollo-8-Mission aufgenommene Bild der Erde einen derartigen Eindruck wie dort. Der 1968-1972 von Steve Jobs herausgegebene „Whole-Earth-Catalogue“ verbreitete dieses Bild auf dem Cover, und Jobs bezeichnete 
den Katalog als die „Bibel meiner Generation“, von der er 2005 noch sagte, er sei „eine Art Google in Taschenbuchformat“ gewesen, dessen Look übrigens im Design der Google-Websites weiterlebt. Wie die Ausstellungsmacher von „The Whole Earth. Kalifornien und das Verschwinden des Außen“, Dietrich Diederichsen und Anselm Franke, anschaulich nachzeichnen, hat die kalifornische Ideologie von Selbstoptimierung, Wellness und Fitness, Technikverehrung und politischen Utopien nicht nur die Welt zu ihrem Logo, sondern verdankt sich in gewisser Weise selbst dem Bild der Welt, bevor sie sich ihr in Form der Globalisierung aufprägen konnte. ${ }^{3}$

Dass der Transhumanismus eine seiner Hochburgen in Kalifornien hat und die Singularity University neben Google steht, ist von daher kaum ein Zufall. Diese Form des transhumanistischen Futurismus könnte man auch als den forcierten Versuch verstehen, die Ideen der abendländischen Metaphysik endlich zu realisieren und den Hegelschen Geist zu vollenden, d.h. ihn als einzig Reales buchstäblich zu verabsolutieren, d.h. abzutrennen von der Endlichkeit seiner Körperlichkeit und ihn unsterblich und unendlich zu machen. Damit wäre der Transhumanismus weniger ein Bruch, als vielmehr der konsequent zu Ende und zur Selbstüberwindung gebrachte Humanismus, der letzte Schritt in der Geschichte der Selbstermächtigung und Selbstschöpfung seit Pico della Miranola, er wäre die Erfüllung aller anthropozentrischen Träume. Den Menschen in seinem Kern zu verändern, sein biologisches Schicksal nach seinem eigenen Bilde und seinen Zwecken zu planen und die Evolution endlich auch der eigenen Gattung nun selbst in die Hand zu nehmen, diesen Auftrag, so verstehen es die Transhumanisten, haben die Menschen von der Natur selbst erhalten, weil die Evolution ihnen das entsprechende Wissen selbst in die Hand gegeben habe. Die organische Neukonstruktion, die eugenische Betreuung der Gattung, die Optimierung der Körper sowie die Eliminierung von Mängeln, die Kopplung des Gehirns an die Maschine, all dies könne man nicht ausschlagen, da die Evolution diejenigen hinter sich ließe, die ihr nicht mehr folgen könnten. Im Klartext und in den Worten von Ray Kurzweil:

„Vor 500 Jahren ist in einem Jahrhundert nicht viel passiert. Heute passiert sehr viel in 6 Monaten. Die Technik nährt sich selbst (!) und ändert sich immer schneller. In etwa 40 Jahren wird sich der Wandel so schnell vollziehen, dass sie nicht in der Lage sein werden ihm zu folgen, außer sie verbessern ihre eigene Intelligenz, indem sie mit der intelligenten Technik, die wir (!) beschaffen, verschmelzen. Das ist so eine tiefgründige Transformation, dass wir eine Metapher aus der Physik entlehnt haben und sie Singularität nennen." (Film; Herv. MW)

Nach Kittler ist es schon längst so weit, dass Menschen den Fortschritten ihrer Wissenschaft und ihren Experimenten nicht mehr folgen können: „Das Wissen von der Natur hat längst aufgehört, wie einst in Königsberg so genannte Erkenntnisse über eine so genannte Erscheinungswelt zu liefern. Was die Physiker an 
Daten noch erreicht, haben computergesteuerte Experimente schon zuvor in Computer und deren Statistikprogramme geschickt. Ganz entsprechend ist das Wissen von den Maschinen in eine Rückkopplungsschleife mit den Maschinen selbst geraten. [...] Das Erwachen aus jenem anthropologischen Schlaf, wie Foucault ihn dem 19. Jahrhundert attestierte, ist jedenfalls kein Menschenwerk." (Kittler 2000, S. 61)

Da klingt es nur konsequent, wenn Peter Diamandis, Mitbegründer der Singularity University, sagt: „Wir werden Gott ähnlich werden. Die Leute hören das nicht gerne, aber unsere Mission ist es, alles zu wissen, unsere Gehirne an Google anschließen zu können, omnipotent zu sein. Etwas auf der anderen Seite der Erde kontrollieren zu können, omnipräsent zu sein. Die Gedanken von jemanden in Japan oder Hawaii zu kennen, von jedem Menschen zu jeder Zeit. Wer dann den Stecker raus zieht wird sehr einsam sein." (Welt ohne Menschen) Und John Smart, Futurologe, Gründer und Präsident der Accelerations Studies Foundation formuliert grundsätzlich:

„Transhumanismus ist die Idee der Verschmelzung von Mensch und Technik. Die Vorstellung, dass der Mensch mehr wird, als er biologisch gesehen ist. Weil er neue Technologien und Wissenschaften nutzt, um sein natürliches biologisches Selbst zu übertreffen. [...] Die meiste Zeit sieht es nicht nach Fortschritt aus. Das ist der Tanz der Evolution. Den tanzen wir, aber unsere Maschinen transzendieren $i h n$, sie wachen auf. Sie sind noch nicht intelligent genug, um Gefühle zu haben. Wir - als ihre Konstrukteure - wissen noch nicht, wie man Maschinen baut, die fühlen können, aber das werden wir irgendwann. Denn wir fangen an zu verstehen, die Mechanismen im Gehirn, die Emotion verursachen, und wenn wir die in unsere Maschinen stecken, werden sie nicht nur denken, sondern auch fühlen. Und wenn sie erwacht sind, werden wir sie nicht mehr als etwas sehen, das von uns getrennt ist, sondern als intime Verlängerung von uns selbst. Wir werden zu ihnen, das ist für mich das wahrscheinlichste Szenario einer Welt jenseits der Menschen. Es ist immer noch Leben. Die Menschheit und alle ihre Elemente sind immer noch vorhanden, aber es ist postbiologisch. Die Maschinen werden in den nächsten 50 Jahren ein Erwachen erleben, ähnlich dem eines Babys, und wenn sie sich zurück erinnern, wie das für sie war, dann ist das ein ziemlich schönes Szenario.“ (Ebd.)

Der Transhumanismus als finale Stufe des Humanismus macht sich anheischig, die letzte „Kadenz der Schöpfung“ (Berr 1994) zu realisieren und den Menschen an die Transzendenz der Maschine anzupassen, denn im Grunde sind biologische Lebewesen für sie schon jetzt nichts anderes als Computer, die mit chemischen Stoffen rechnen. Der Geist als kopierbares Programm oder speicherbare Software, das individuelle Bewusstsein und Gedächtnis als die Gesamtheit aller Synapsen eines Gehirns, das mittels Nanobots gescannt und auf entsprechende Hardware übertragen und gespeichert oder implementiert werden kann, diese in den Bereich 
des Möglichen eingedrungenen Visionen könnte man verwechseln mit einer Versöhnung von Technik und Humanismus. Doch folgt man den Überlegungen Heideggers ([1946] 1978), gehören beide schon lange dem Raum der Metaphysik an, da die humanistische Weltbemächtigung und das neuzeitliche technische Weltverhältnis schon immer eine Einheit gebildet hätten. So kann man auf den Transhumanismus auch kaum dadurch antworten, dass man sich auf das „eigentliche“ Wesen des Humanismus beruft. Wie Heidegger schon in seinem „Brief über den ,Humanismus" "schrieb, müsse man das Wort Humanismus aufgeben, weil er das Wesen des Menschen gar nicht erfasst habe. Er könne nicht als Lösung in Anspruch genommen werden, da der „Mensch selbst mitsamt seinen Systemen metaphysischer Selbstüberhöhung und Selbsterklärung das Problem ist“ (Sloterdijk 1999, S. 23). Worum es gehen müsse, wäre die „Humanitas des homo humanus“ (Heidegger 1978, S. 341) anfänglicher zu denken. Da dieses andere Verständnis des Menschen jedoch ,gegen allen bisherigen Humanismus spricht, aber sich ganz und gar nicht zum Fürsprecher des Inhumanen macht" (ebd., S. 342), müsse das Wort aufgegeben werden. Man muss also dem Humanismus entkommen, der die Frage nach dem Menschen glaubt beantwortet zu haben und alle andere Antworten nur als unmenschlich qualifizieren kann. Dagegen käme es darauf an, den Menschen und seine Menschlichkeit anders zu denken als im humanistischen Schema, das die Ideologie der Unmenschlichkeit ist, da alles, was sich der Wesenbestimmung nicht fügt, als unmenschlich qualifiziert und behandelt wird.

\section{Posthumanismus}

Die technikzentrierte Vorstellungen einer Überwindung des Menschen entsprechen in weiten Teilen dem kulturellen Imaginären, wie es sich in der Populärkultur von Science Fiction und vor allem in Filmen, PC- und Videospielen manifestiert, wo die Virtualisierung, Cyborgisierung, Hybridisierung und Maschinisierung fast schon als etwas Normales inszeniert wird. Aber auch in der allgegenwärtigen und von Žižek so genannten „dritten Kultur“, einem Produkt der „,kognitivistischen Popularisierer der hard sciences " ${ }^{\star 4}$, entsprechen diese transhumanistischen Phantasien weit verbreiteten Ansichten über die mögliche Zukunft des Menschen, die in der Regel so dargestellt wird, als ob sie von einem technologischen Determinismus beherrscht wäre, wobei einem technophilen Optimismus meistens ein technophober Kulturpessimismus korrespondiert, die Diskussion also vollständig im Raum des humanistischen Denksystems verbleibt.

Dabei drängt sich eine Vielzahl von kritischen Nachfragen geradezu auf, die unterschiedliche Ebenen betreffen: Technologisch realisierbar sind diese Visionen sicher noch nicht, noch sind es also Visionen. Doch wenn auch schon vieles 
möglich ist - die künstlich erschaffte Zelle, das Klonen von Menschen, die genetische Manipulation, die Verbindung zwischen Zellen und nichtorganischen Substanzen, die Neukonstruktion der Materie durch den Zugriff auf einzelne Atome, die Nutzung von Gehirnströmen zur Steuerung von Computern - könnte man eines Tages wirklich das Gehirn als ganzes scannen? Könnte man ohne Körper denken? (Lyotard 1989) Liegt die Bedeutung des Transhumanismus aber überhaupt in seinen Utopien? Liegt seine Wirksamkeit nicht viel mehr im Politischen, insofern sich vermittelt über und verbunden durch die Phantasmen ein elitäres und bereits sehr machtvolles Netzwerk aus Militär, Rüstungsindustrie, Politik, IT-Konzernen und wissenschaftlichen Forschungsinstitutionen gebildet hat? Und läuft das alles nicht auf eine Spaltung der Gesellschaft hinaus, auf einen Krieg gegen diejenigen ohne Zugang zu den Technologien, auf eine ultrareaktionäre Version der Radikal-Libertarier in der Nachfolge von Ayn Rand, wie Jean-Paul Malrieu, Quantenphysiker und CNRS Forschungsdirektor vermutet? „Der Zugang zu immer höher entwickelter und kostspieligerer Technik führt nicht unbedingt zu einer Verbesserung des Gemeinwohls. Das kann genauso das Projekt einer Elite sein und somit einen gewaltigen Bruch in der Gesellschaft verursachen. Das stellt ein politisches Problem dar und zwar, wie ich meine, eines der schwerwiegendsten für die heutige Menschheit. Diese Leute lassen gerne einen beträchtlichen Teil der Menschheit am Straßenrand stehen, bis er endgültig verschwindet. Es bedarf dazu keiner wirklichen Vernichtung, sie werden ausgelöscht, weil sie weniger Wert sind, das wird ihnen zum Verhängnis. Auf gewisse Weise wird so eine Art Zäsur, ein Bruch hingenommen." (Film)

So berechtigt diese Fragen sind, es bedarf einer gewissen Vorsicht. Denn um die transhumanistischen Visionen anders beurteilen zu können, aus einer Perspektive, die eine Kritik ermöglicht, die die Geltung der vom Humanismus vorgegebenen Kriterien und Abgrenzungen in ihre Kritik einbezieht, muss man der eben angedeuteten Alternative zwischen futuristischem Technikfetischismus und konservativem Humanismus entkommen. Versuche dieser Art möchte ich hier unter dem Namen eines dekonstruktiven Posthumanismus ansprechen, ohne zu behaupten, hier ließe sich eine Einheit unter den Autoren, den Ansätzen und Philosophien erkennen, die vielmehr untereinander eine heterogene Vielheit bilden, die aber dennoch Berührungspunkte aufweisen. Der Posthumanismus also ist kurz gesagt zu verstehen als ein Versuch, das Humanum neu und anders zu denken, ohne der apokalyptischen Mystik, neuen Formen des Spiritismus oder technizistischen Utopien zu verfallen. Das Ende des Menschen denken heißt dann, die mit ihm konnotierten Illusionen und die in seinem Namen realisierte Geschichte kritisch durchzuarbeiten oder zu dekonstruieren, um zu verhindern, dass aus der „Introversion des Opfers“ (Horkheimer/Adorno 1968) ein vollständiges Selbstopfer wird, dass die Beseitigung aller natürlichen Mängel aus dem Menschen ein 
Monstrum macht und die Verwirklichung der Vollendungsutopien in einem realisierten Albtraum endet.

So besteht die Problematik von Vervollkommnungsprogrammen darin, dass sie von unverbesserlichen Menschen unternommen werden, die in der Regel nicht erkennen, dass ihre Vervollkommnungsideen Symptome ihrer Unvollkommenheit sind. Um noch einmal Kamper zu zitieren: „Was vorschwebt, ist die Vision, Menschen nach dem Bilde vollkommener Menschen zu erzeugen und zu züchten. $\mathrm{Da} ß$ man es könnte, ist ein unverbesserliches Mißverständnis. Denn die Unverbesserlichkeit der Täter wird intervenieren.“ (Kamper 1994, S. 276) Mit andern Worten ist der Traum von der Überwindung der Unverbesserlichkeit selbst Teil der Unverbesserlichkeit. Das Leiden aus der Welt schaffen, den Tod überwinden wollen, unverletzlich werden, alles Übel austreiben, alles Bedrohliche, Unangenehme, Widerständige, Fremde vernichten - man muss das nicht weiterführen, um die nihilistische Bahn und den Abgrund zu erkennen, in den dieser Traum mündet.

Das gilt nicht nur für alle und alles, was diese Gewalt der Vervollkommnung erfassen mag, sondern auch für den sich optimierenden Menschen selbst. Denn der Traum der Unsterblichkeit verspricht keineswegs ein ewiges Glück, wie u.a. Baudrillard sehr deutlich gemacht hat. Mit Blick auf „Biosphäre 2“ in Arizona, einer Simulation der Erde mit dem Zweck, die Überlebensbedingungen zu erforschen, schreibt er z.B.: „Der wirkliche Planet wird im voraus seinem miniaturisierten, klimatisierten Klon geopfert, der vermöge der totalen Simulation den Tod besiegen soll. Einstmals waren es die Toten, die man für die Ewigkeit einbalsamierte; heute sind es die Lebenden, die lebendig in das Überleben einbalsamiert werden.“ (Baudrillard 1994, S. 337) Unsterblichkeit kann für Menschen nur heißen, den Tod überleben, und da es mehrere Formen des Todes gibt, ist keineswegs klar, ob das Leben danach noch das Leben wäre, das man verewigen wollte, oder ob dies mit ausgelöscht würde: „Man kann den Tod auch auslöschen, indem man unzerstörbare Lebensprozesse erschafft; genau das, was wir tun, wenn wir die Unsterblichkeit in den anatomischen, biologischen und genetischen Prozessen zu erschleichen versuchen.“ (Ebd., S. 350) Die zu erwartende moderne Unsterblichkeit wäre nicht die alte, religiöse Überlebendigkeit des ewigen Lebens, sondern „eine Qualität des Über-Lebenden, das heißt desjenigen, was bereits tot ist und aus diesem Grunde unsterblich wird, aber keineswegs mehr in gleicher Weise. [...] [Es ist die Qualität dessen,] was kein Ende mehr kennt, da es bereits über sein eigenes Ende, seine eigenen Ziele hinausgegangen, jenseits seiner eigenen Möglichkeiten und in gewisser Weise in Hypertelie oder ins Koma übergegangen ist.“ (Ebd., S. 351)

Diese Andeutungen mögen genügen, um die Problematik transhumanistischer Projektionen deutlich zu machen, aber auch die Perspektive des Posthumanismus. Dieser muss die technologischen Herausforderungen annehmen, und zwar nicht 
nur, weil die fortschreitende Technologisierung der Spezies Mensch nicht zu leugnen ist, sondern weil es das Humane nicht ohne technische Bedingungen gäbe und diese nicht erst später hinzutreten, sondern von Beginn an bei seiner Konstitution mitwirken und ihm auch nicht äußerlich bleiben, sondern mit dem zur Unsichtbarkeit verschmelzen, was er sein Eigenstes nennen wird, angefangen mit seinen Erinnerungen, seinem Gedächtnis, seinem Denkvermögen, seiner Subjektivität bis hin zu seiner Sprache. Das vermeintlich Eigene als etwas ursprünglich Fremdes zu erkennen, das erst zu etwas Eigenem wurde, das ist die Gegenbewegung zum humanistischen Entfremdungsdiskurs, der unter dem Schein des Fremden immer etwas ursprünglich Eigenes vermutet oder etwas Feindliches, von dem man sich befreien muss. Das Technische ist damit nicht das Gegenteil des Humanen, sondern gehört zu seinem Kern, der allerdings nie ein Wesens-Kern gewesen sein wird, sondern von Anfang an als Spaltung vorgestellt werden müsste, als eine Leerstelle, die nachträglich von einem Supplement in Gestalt eines Pharmakon markiert worden sein wird, das sich zu etwas hinzufügt, was es noch gar nicht gibt, sondern durch diese Supplementierung erst entsteht. Technik als das „Inhumane" (Lyotard 1989), das Heterogene, Fremde, Andere hat den Menschen heimgesucht und gehört zu ihm wie die Sprache und sein Unbewusstes. Ihm verdankt der Hominide seine Humanität.

Der Posthumanismus versucht, dieses Humane im Sinne einer Differenz statt als Gegensatz zur und mit dem Inhumanen, d.h. der Technik, aber auch zum und mit dem Tier, zur und mit der Maschine, zum und mit dem Anderen zu denken. Es geht mithin darum, etwas Ungedachtes zur Sprache zu bringen. Dieses Ungedachte, diese Leerstelle und Unbestimmtheit ist einerseits Ausgangs- und Angriffspunkt der Pädagogik wie auch der Entwicklungs- und Optimierungsideologie der liberalen Marktlogik, für die der Mensch nur Mittel zum Zweck ist, und natürlich auch für das transhumanistische Schöpfungsprogramm. Sie ist aber andererseits genau der in der Unterscheidung von Natur und Kultur nicht aufgehende Rest, der aus der dualistischen Ontologie und dem metaphysischen Humanismus ausgeschlossen wird und verantwortlich dafür ist, dass der Mensch ein Wesen ist, das kein Wesen hat, das von Natur aus von Natur frei ist, dessen Wesen also in dessen Abwesenheit besteht: „Es genügt unseren Zeitgenossen im Grunde daran zu erinnern, daß das Eigentliche des Menschen sein Mangel an Eigentlichem ist, sein Nichts oder seine Transzendenz, um , ausgebucht' zu sein.“ (Lyotard 1989, S. 16) Die Ek-zentrizität des Menschen, seine Plastizität und Unbestimmtheit verlangt daher eine doppelte Analyse, die ihn zum einen als historisch-kulturelles Resultat seiner eigenen Geschichte begreift, die aber zum anderen die Spuren zum Vorschein bringt, die der Andere und das ihm Heterogene in ihm hinterlassen haben.

So weist z.B. Derrida in seinem Text „Les Fins de l'homme“ von 1968 entschieden den Hegelianismus eines Denkens des Endes des Menschen zurück, das 
immer in einer teleologischen Aufhebung eines ,ich“ oder „wir“ mündet, wie z.B. in einem „Sein zum Tode“. Statt dessen geht es ihm um ein Denken des Humanen als radikale Öffnung auf den Anderen, auf das „Unmenschliche“ der Schrift als seiner technologischen Bedingung wie auch des Tieres und auf das Unmögliche der Zukunft. Wenn der extremste Ausdruck der Anthropologie das Zeitalter der Technik ist, wie Nancy schrieb (1981, S. 13), dann führt dahin nur eine Dekonstruktion des humanistischen Verhältnisses Mensch-Technik, um die unmögliche Möglichkeit eines ganz anderen Menschen denkbar zu machen, postmetaphysische Subjektivitäten auch nicht-menschlicher Wesen und plurale Singularitäten im Sinne Nancys (Nancy 2004).

Das, was nach all den Dekonstruktionen des Subjekts übrig geblieben ist, das es „nie für irgendjemanden gegeben hat“, weil es „ein Märchen ist“, das allerdings sehr ernst genommen werden müsse (Derrida in Cadava 1991, S. 102), dieser Rest besteht im Wesentlichen in dem, was in humanistischer Perspektive mit der Schwäche, der Unvollkommenheit und der Unverbesserlichkeit des Menschen zu tun hatte: seine Unbestimmtheit, Temporalität, Endlichkeit, seine Unidualität (Morin 1994) und Selbstfremdheit, seine Körperlichkeit und Verletzlichkeit, seine Plastizität, Offenheit und Unberechenbarkeit. Diese Begriffe bezeichnen in Form der Negativität das, was als Positivität zu verstehen wäre, was aber in einer Positivierung sofort in Gefahr stünde, in die Fallen des anthropologischen Denkens zurück zu führen. Jeremy Rifkin, Ökonom, Publizist, Gründer und Vorsitzender der Foundation on Economic Trends verdeutlicht das Gemeinte sehr vereinfacht, doch auch recht anschaulich: „Ich glaube, wenn wir uns auf die Unsterblichkeit konzentrieren und darauf, technische Ersatzmittel zu nutzen, um das zu ersetzen, was wir haben, dann verlieren wir die Essenz dessen, was uns zu Menschen macht - unsere Schwächen, unsere Fehler. Ich wäre ziemlich nervös, müsste ich in einer Welt leben, in der wir nach Perfektion streben. Wir neigen eher dazu, jemanden gegenüber intolerant zu sein, der unser Level nicht erreicht, der nicht perfekt ist, der den technischen Standards, die wir setzen, nicht gewachsen ist. Welche Standards sind das? Qualitätskontrolle, Vorhersagbarkeit der Ergebnisse, ein hohes Maß an Effizienz und Nutzen. Wollen wir in einer Welt leben, in der Menschen nur nach diesen Einheiten gemessen werden, dann werden wir zu Maschinen.“ (Film)

Eine Chance, der Utopie der Vervollkommnung als Symptom der Unverbesserlichkeit zu entkommen, wäre die posthumanistische Einsicht darin, dass dieses Bestreben ein Symptom ist und das Heilmittel nicht im Verschwinden des Menschen durch eine Überdosis Technik und einer Verschmelzung mit der Maschine besteht. Die Mängel abzuschaffen hieße nämlich nicht Vervollkommnung, sondern liefe auf die Vernichtung des Humanen hinaus. Oder wie Dietmar Kamper es sagt: „Jene Menschen nämlich, die den Weg der Vervollkommnung weit genug 
gegangen sind, denken nicht im entferntesten daran, mittels der Technologie die Verhältnisse zu verbessern. Ihr Ja zum Leben der Gattung schloß immer auch deren Unverbesserlichkeit ein. Vielleicht besteht Vervollkommnung in nichts anderem als in der schrittweise geleisteten Einsicht in die Unabdingbarkeit des Nicht-Vollkommenen: der Gebrechlichkeit, der Hinfälligkeit, der Sterblichkeit der menschlichen Natur.“ (Kamper 1994, S. 276)

\section{Bildung - was sonst?}

Wie kann man nach all diesen Demontagen des humanistischen und anthropozentrischen Selbstverständnisses noch von Bildung sprechen? Ist es nicht aus allen Gründen endgültig vorbei mit der Bildung, sowohl als Begriff wie auch als Versprechen oder gar als „le travail de soi sur soi“ bzw. als Praxis der Selbstsorge? Und wenn Bildung ihr Ende überlebt oder bereits ins Stadium des einbalsamierten Überlebens eingetreten und in gewisser Weise zu etwas Untotem geworden ist, zu einem Gespenst, das aber im Koma liegt, sollte man sie dann nicht besser von ihrem Elend erlösen, statt ihr neues Blut einzuflößen oder einen Herzschrittmacher einzupflanzen? Bisher sind aber alle Versuche, sie durch andere Begriffe zu ersetzen, gescheitert, wohingegen ihre Beharrungskraft abnimmt und ihr Sinngehalt immer undeutlicher und diffuser wird, je mehr sie zum Zentrum im Diskurs um die Zukunft wird und je intensiver man sie befördern will. Als würde sie nur im Dunklen leuchten, verliert sie ihren Reiz, sobald man sie dem Licht von Klassenzimmern aussetzt. Vielleicht hatte sie schon immer diesen irrlichternden Glanz, von dem Nietzsche vermutete, er sei schon alles, und vielleicht gehorcht sie einer Gespensterlogik, deren Aufklärung und Berechnung ihrer Auftretenswahrscheinlichkeit man von der empirischen Bildungsforschung wohl kaum erwarten kann.

Dies zu klären habe jedoch auch ich hier und jetzt nicht die Zeit. Illusorisch jedoch scheint mir, dass man sich vom Bildungsdiskurs einfach dadurch befreien könnte, dass man behauptet, es gäbe sie nicht mehr, sie wäre eine Gestalt der Vergangenheit und man solle daher auch auf das Wort verzichten. Ob es allerdings gelingen kann, dieses leer gewordene Wort neu zu füllen oder es in den Dienst seiner eigenen Autodekonstruktion zu stellen, ist eine keineswegs bereits entschiedene Frage. Es käme auf Versuche an, die durchaus aus verschiedenen Perspektiven heraus erfolgen können. Abschließend möchte ich noch wenigstes die Richtung andeuten, in die man dabei gehen könnte.

Wie ich zu zeigen versucht habe, ist trotz des Diskurses über das Ende des Menschen die Frage nach dem Humanen keineswegs obsolet geworden. Zwar haben die Menschen ihre Sonderstellung in der Schöpfung, im Kosmos und in der Natur verloren, sie haben die Kränkungen von Kopernikus, Darwin, Freud, Turing 
und der Gentechnik erfahren, durch die sie aus dem Mittelpunkt des Universums vertrieben wurden, erst ihren göttlichen Ursprung und dann die Herrschaft über sich selbst verloren, bevor sie ihr vermeintliches Alleinstellungsmerkmal mit einer Maschine teilen mussten und auch ihre biologische Spitzenstellung auf einen minimalen genetischen Abstand zu den Schimpansen (98,4\% Identität) zusammen geschmolzen ist. Aber all diese Kränkungen und Enttäuschungen haben nur gezeigt, dass die Menschen mit ihren Selbstbildern und -definitionen nicht identisch, aber auch nicht Nichts, sondern immer auch noch anders sind und sein können. Es ist dieser „Fehler des Epimetheus“ (Stiegler 2009a, S. 46ff; 2009b), der von der traditionellen Anthropologie nicht als etwas positives, sondern dogmatisch als Mangel angesehen wurde, der aber die Bindungen der Menschen untereinander wie auch zur Welt erst ermöglicht.

Für jedes weitere Nachdenken über Bildungsprozesse bedeuten diese Überlegungen, dass alle Konzepte und Theorien nicht mehr anschlussfähig sind oder dekonstruiert werden müssten, die offen oder implizit teleologisch sind. Darunter fallen alle Ziel- und Zweckbestimmungen des Menschen (Fichte) und der Bildung (Humboldt), insbesondere alle Arten von Bildungskonzepten, die Bildung als Selbstbildung begreifen, als selbstgesteuert, selbstverantwortet, frei und selbstbestimmt, die den Menschen als autopoietisches System und Kinder als kleine Lernautomaten und kybernetische Maschinen betrachten. Auch diejenigen Bildungstheorien fallen darunter, die in Bildung noch das Echo eines Versöhnungsversprechens zu vernehmen glauben, eine Vereinigung von Individuum und Gesellschaft oder von Natur und Kultur, oder wenigstens den Gewinn einer individuellen Freiheit und Urteilskraft, die in den Dienst von Aufklärung und Emanzipation gestellt werden könnten. Das heißt nun nicht, dass Humboldts Bedingungen für Bildungsprozesse, Freiheit und Mannigfaltigkeit, und seine Vorstellungen einer explorativ-experimentellen Exprimierung der eigenen Möglichkeiten durch entdeckende Erfahrungen (Ahrens 2011) verworfen würde. Nur stehen diese Prozesse nicht mehr im Dienste der freien Entfaltung der Persönlichkeit und der Steigerung der Individualität als Zweck des Menschen, sondern sind als Antworten auf das Reale, das radikale Anderssein der Anderen und der Welt zu verstehen.

Was erforderlich ist, wäre also ein dekonstruktiver Perspektivwechsel, für den man an der dem Bildungsbegriff inhärenten Ambivalenz ansetzen kann. Einerseits wird ja in der Regel mit dem Bildungsbegriff ein funktionaler Systemzusammenhang gekennzeichnet. Andererseits dient der Bildungsbegriff als Bezugspunkt der Kritik genau an diesem Bildungssystem. Und so wie dieser Begriff mit sich nicht identisch ist, so wenig sind es die sich bildenden Individuen. Auch die aktuelle Optimierungs- und Steigerungslogik von Bildung vermag es nicht, die Individuen mit einer bruchlosen Identität und einem unproblematischen Selbstbezug auszustatten. Der Begriff der Bildung verweist nämlich auch in seinen funktionalen 
Verkürzungen immer auf Erfahrungen, die das Subjekt an seine Grenzen führen und mit Negativität, unaufhebbarer Differenz, Alterität und Fremdheit konfrontieren. So vollziehen sich Bildungsprozesse wohl immer in einem Spannungsfeld zwischen Erfahrung als Widerfahrnis, Leidenschaft, Passivität einerseits und Aktivität, Selbstbestimmung, Autonomisierung andererseits, zwischen dem Gesetz der Gabe und der Gabe des Gesetzes.

Wie Heydorn sagte, geht es mit Blick auf die gegenwärtigen technologischen Entwicklungen und ihren psychischen, kulturellen, gesellschaftlichen wie auch globalen Ausprägungen darum, „das Bewußtsein des Menschen von sich selber auf die Höhe der technologischen Revolution zu bringen." Aber dies würde eben nicht bedeuten, sich wieder zum obersten Steuermann zu machen (zum

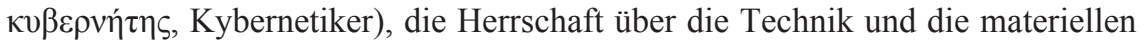
Bedingungen zurück zu erobern und das anthropozentrische Werk der Weltbemächtigung fortzusetzen und zu Ende zu führen, wie dies Heydorn vorschwebte. Statt auf diesem Weg der Selbst- und Weltbeherrschung weiter zu gehen oder das Unmögliche einer vollkommenen Herrschaft über die technologischen Bedingungen im Sinne einer Befreiung von ihnen realisieren zu wollen, ginge es wohl in erster Linie darum, leben zu lernen und dass wir uns fragen, wie wir leben wollen. Womit man deshalb auf jeden Fall beginnen müsste, wäre in der Tat ein Kampf um die Lebensbedingungen. Oder wie es Roland Gori, Professor für Psychologie und klinische Psychopathologie, im Film sagte: „Wir müssen damit aufhören, unser Leben von Experten bestimmen zu lassen. Ob in der Wirtschaft, der Politik, der Nanotechnologie oder wo auch immer. Wir müssen die Demokratie zurück erobern, die wir ihnen unter dem Druck der Sicherheitspolitik und verschiedener Humanindustrien beinahe überlassen haben.“

\section{Anmerkungen}

1 Der von Bouglé 1905 in die Soziologie eingeführte Begriff „Posthistoire“ meint ,jenen gesellschaftlichen Zustand des Vergessens bzw. des Nichtmehr-Wartens, den die Apokalyptiker am meisten von allen Seelenhaltungen gefürchtet haben. Es geht um den ,etat final' der fortgeschrittenen Gesellschaft, die all ihre Spannungen im Prinzip aufgelöst hat, sie bestenfalls noch simuliert, um Gewohnheiten beibehalten zu können [...] die Gesellschaft funktioniert präzise wie eine Maschine, nicht trotz, sondern wegen der Störungen, die gewinnbringend umgesetzt werden; die Krise [...] ist institutionell auf Dauer gestellt und neutralisiert längst jede Form von Kritik [...] Das Posthistoire kann somit als Gegenteil der Apokalypse gelten: was bei dieser durch Offenbarung in Erscheinung tritt, verschwindet bei jenem durch Zerstreuung und Vergessen; so hochdramatisch diese sich inszeniert, so langweilig und spannungslos fällt jenes aus; während die Eschatologie von der Eskalation der Katastrophen berichtet und sie immer wieder in Erinnerung ruft, hält das Posthistoire es mit der Routine, die 
Neues nur zuläßt, wenn es aus dem Fundus des Uralten stammt, und die auf Innovation jeglicher Art das Mittel der paradoxen Neutralisierung anwendet.“ (Kamper 1986, S. 63f)

2 Der Dokumentarfilm von Philippe Borrel entstand 2012 in Frankreich und wurde am 23. Oktober 2012 in Arte ausgestrahlt. Gezeigt werden in verschiedenen Bereichen die neuesten technischen Möglichkeiten für Menschen mit Behinderungen, aber auch für die ,Verbesserung“ nicht-behinderter Menschen, auf die sich das transhumanistische Projekt als empirische Beweise der Realisierbarkeit seiner Utopie stützt, deren sehr elitäre Ansichten und Bestrebungen motiviert werden durch ein unverkennbar ebenso religiöses wie biopolitisches Phantasma.

3 Vgl. dazu den vom Haus der Kulturen der Welt in Berlin herausgegebenen Katalog zur Ausstellung vom 26. April bis 1. Juli 2013 (Diederichsen/Franke 2013).

4 Eine Mischung aus Feuilleton, Wissenschaftsmagazinen, Futurologen, Marketingbranchen, Lobbyisten etc. Vgl. Herbrechter (2009, S. 19f).

\section{Literatur und Film}

Adorno, Theodor W.: Eingriffe, Frankfurt am Main 2003.

Adorno, Theodor W.: Theorie der Halbbildung. In: ders.: Gesammelte Schriften Band 8. Soziologische Schriften I, Frankfurt am Main 1997, S. 93-121.

Ahrens, Sönke: Experiment und Exploration. Bildung als experimentelle Form der Welterschließung, Bielefeld 2011.

Altner, Günther: Leben in der Hand des Menschen, Darmstadt 1998.

Anders, Günther: Die Antiquiertheit des Menschen, 2 Bde., München 1980 u. 1981.

Baudrillard, Jean: Überleben und Unsterblichkeit. In: Kamper/Wulf 1994, S. 335-356.

Bedorf, Thomas/Joachim Fischer/Gesa Lindemann (Hg.): Theorien des Dritten, München 2010.

Berr, Marie-Anne: Die Kadenzen der Schöpfung: Gott-Mensch-Maschine. In: Kamper/ Wulf 1994, S. 203-215.

BMBF (Hg.): Expertise: Zur Entwicklung nationaler Bildungsstandards, Berlin 2003.

Böschen, Stafan/Michael Schneider/Anton Lerf (Hg.): Handeln trotz Nichtwissen, Frankfurt/New York 2004.

Bolz, Norbert: Weltkommunikation, München 2001.

Cadava, Eduardo (Hg.): Who Comes After the Subject? New York 1991.

Coenen, Christopher/Stefan Gammel /Reinhard Heil/Andreas Woyke (Hg.): Die Debatte über „Human Enhancement“, Bielefeld 2010.

Derrida, Jacques: Fines hominis. In: Randgänge der Philosopohie, Wien 1988, S. 119-142.

Diederichsen, Dietrich/Anselm Franke (Hg.): The Whole Earth. Kalifornien und das Verschwinden des Außen, Berlin 2013.

Eßlinger, Eva/Tobias Schlechtriemen/Dori Schweitzer/Alexander Zons (Hg.): Die Figur des Dritten. Ein kulturwissenschaftliches Paradigma, Frankfurt am Main 2010.

Gamm, Gerhard: Der unbestimmte Mensch. Zur medialen Konstruktion von Subjektivität, Berlin/Wien 2004.

Gustafsson, Lars: Utopien, Frankfurt/Berlin/Wien 1985.

Habermas, Jürgen: Die Zukunft der menschlichen Natur. Auf dem Weg zu einer liberalen Eugenik? Frankfurt am Main 2001. 
Hagner, Michael/Erich Hörl (Hg.): Die Transformation des Humanen. Beiträge zur Kulturgeschichte der Kybernetik, Frankfurt am Main 2008.

Haraway, Donna: Ein Manifest für Cyborgs. Feminismus im Streit mit den Technowissenschaften. In: Die Neuerfindung der Natur. Frankfurt/New York 1995, S. 33-72

Hardt, Michael/Negri Antonio: Empire. Die neue Weltordnung, Frankfurt am Main 2002.

Heidegger, Martin: Die Zeit des Weltbildes (1938). In: Holzwege. Frankfurt am Main 1980, S. 73-110.

Heidegger, Martin: Brief über den Humanismus (1946). In: Wegmarken, Frankfurt am Main 1978, S. 311-360.

Heydorn, Heinz-Joachim: Zu einer Neufassung des Bildungsbegriffs, Frankfurt am Main 1972.

Herbrechter, Stefan: Posthumanismus. Eine kritische Einführung, Darmstadt 2009.

Horkheimer, Max/Theodor W. Adorno: Dialektik der Aufklärung, Amsterdam 1968.

Jonas, Hans: Technik, Medizin und Ethik, Frankfurt am Main 1987.

Kamper, Dietmar: Zur Soziologie der Imagination, München 1986.

Kamper, Dietmar: Unmögliche Gegenwart. Zur Theorie der Phantasie, München 1995.

Kamper, Dietmar: Der eingebildete Mensch. In: Kamper/Wulf 1994, S. 273-278.

Kamper, Dietmar/Christoph Wulf (Hg.): Anthropologie nach dem Tode des Menschen, Frankfurt am Main 1994, S. 7-14.

Kittler, Friedrich A. (Hg.): Austreibung des Geistes aus den Geisteswissenschaften, Paderborn/München/Wien/Zürich 1980.

Kittler, Friedrich A.: Von der Zukunft des Wissens. In: 7 Hügel - Bilder und Zeichen des 21. Jahrhunderts, VI) Wissen, Berlin 2000, S. 59-61.

Klafki, Wolfgang: Konturen eines neuen Allgemeinbildungskonzepts. In: Neue Studien zur Bildungstheorie und Didaktik, Weinheim/Basel 2007, S. 43-81.

Koller, Hans-Christoph: Bildung anders denken. Einführung in die Theorie transformatorischer Bildungsprozesse, Stuttgart 2012.

Kopacek, P.: Menschliche Roboter - Robotermenschen. In: Schmidinger/Sedmak 2004, 113-123.

Latour, Bruno: Wir sind nie modern gewesen. Versuch einer symmetrischen Anthropologie, Frankfurt am Main 1998.

Leibniz, Gottfried Wilhelm: Monadologie, Stuttgart 1979.

Levinas, Emmanuel: Humanismus des anderen Menschen, Hamburg 1989.

Levinas, Emmanuel: Jenseits des Seins oder anders als Sein geschieht, Freiburg/München 1992.

Liessmann, Konrad Paul: Theorie der Unbildung, Wien 2006.

Lohmann, Ingrid/Rainer Rilling (Hg.): Die verkaufte Bildung, Opladen 2002.

Luhmann, Niklas: Das Recht der Gesellschaft, Frankfurt am Main 1998.

Lyotard, Jean-François: Das Inhumane, Wien 1989.

Lyotard, Jean-François: Ob man ohne Körper denken kann. In: Das Inhumane, Wien 1989, S. 23-50.

Maset, Pierangelo: Geistessterben. Eine Diagnose, Stuttgart 2010.

Masschelein, Jan/Norbert Ricken: Do We (Still) Need the Concept of Bildung? In: Educational Philosophy and Theory, Special Issue „Bildung“, Vol. 35, 2003, No. 2, S. 139-154.

Masschelein, Jan/Maarten Simons: An Adequate Education in a Globalised World? A Note on Immunisation Against Being-Together. In: Journal of Philosophy of Education, Vol. 36, No. 4, 2002, S. 589-608. 
Mayer, Ralf/Christiane Thompson/Michael Wimmer (Hg.): Inszenierung und Optimierung des Selbst. Zur Analyse gegenwärtiger Selbsttechnologien, Wiesbaden 2013.

Meyer, Torsten/Wey-Han Tan/Christina Schwalbe/Ralf Appelt (Hg.): Medien \& Bildung, Wiesbaden 2011.

Morin, Edgar: Die Unidualität des Menschen. In: Kamper/Wulf 1994, S. 15-24.

Nancy, Jean-Luc/Philipp Lacoue-Labarthe (Hg.): Les fins de l'homme - á partir du travail de Jacques Derrida, Paris 1981.

Nancy, Jean-Luc: Philosophie und Bildung. In: Bolz, Norbert (Hg.): Wer hat Angst vor der Philosophie? Paderborn u.a. 1982, S. 221-241.

Nancy, Jean-Luc: singuär plural sein, Berlin 2004.

Nietzsche, Friedrich: Unzeitgemäße Betrachtungen. Zweites Stück: Vom Nutzen und Nachtheil der Historie für das Leben. In: Colli, G./Montinari, M. (Hg.): Kritische Studienausgabe, Bd. I, München/Berlin/New York 1988a, S. 243-334.

Nietzsche, Friedrich: Morgenröte. In: Kritische Studienausgabe, Bd. III, München/Berlin / New York 1988b, S. 9-323.

Nowotny, Helga: Eigenzeit, Frankfurt am Main 1993.

Pongratz, Ludwig A.: Bildung im Bermuda-Dreieck: Bologna - Lissabon - Berlin: Eine Kritik der Bildungsreform, Paderborn 2009

Pongratz, Ludwig A.: Sackgassen der Bildung. Pädagogik anders denken, Paderborn 2010.

Rieger, Stefan: Kybernetische Anthropologie. Eine Geschichte der Virtualität, Frankfurt am Main 2003.

Rößer, Barbara: Wissensgesellschaftliche Pädagogik. Der transformierte Bildungsdiskurs als Realisierungs- und Ideologisierungsform der Wissensgesellschaft. In: Bittlingmayer, Uwe H./Ullrich Bauer (Hrsg.): Die Wissensgesellschaft. Mythos, Ideologie oder Realität? Wiesbaden 2006, S. 251-284.

Ruhloff, Jörg: Bildung heute. In: Pädagogische Korrespondenz, H. 21, 1997, S. 23-31.

Ruhloff, Jörg: Lernfabrik oder Bildungsschule? In: Heitger, Marian (Hg.): Wozu Schule? Innsbruck/Wien 2002.

Sattler, Elisabeth: Die riskierte Souveränität, Bielefeld 2009.

Schäfer, Alfred: Das Bildungsproblem nach der humanistischen Illusion, Weinheim 1996.

Schäfer, Alfred: Das Versprechen der Bildung, Paderborn u.a. 2011.

Schirrmacher, Frank (Hg.): Die Darwin AG. Wie Nanotechnologie, Biotechnologie und Computer den neuen Menschen träumen, Köln 2001.

Schöller, Oliver: Bildung geht stiften. Zur Rolle von Think Tanks in der Wissensgesellschaft. In: Bittlingmayer, Uwe H./Ullrich Bauer (Hrsg.): Die Wissensgesellschaft. Mythos, Ideologie oder Realität? Wiesbaden 2006, S. 285-320.

Schroer, Markus (Hg.): Soziologie des Körpers, Frankfurt am Main 2005.

Sloterdijk, Peter: Regeln für den Menschenpark, Frankfurt/Main 1999.

Stiegler, Bernard: Denken bis an die Grenzen der Maschine, Zürich/Berlin 2009a.

Stiegler, Bernard: Technik und Zeit. Der Fehler des Epimetheus, Zürich/Berlin 2009b.

Stiegler, Bernard: Allgemeine Organologie und positive Pharmakologie. In: Hörl, Erich (Hg.): Die technologische Bedingung, Frankfurt am Main 2011, S. 110-146.

Thompson, Christiane/Gabriele Weiß (Hg.): Bildende Widerstände - widerständige Bildung, Bielefeld 2008.

Turing, Alan: Intelligence Service. Schriften, hrsg. v. Bernhard Dotzler u. Friedrich A. Kittler, Berlin 1987.

Villa, Paula-Irene (Hg.): schön normal. Manipulationen am Körper als Technologien des Selbst, Bielefeld 2008. 
Wehling, Peter: Im Schatten des Wissens? Perspektiven der Soziologie des Nichtwissens, Konstanz 2006.

Welsch, Wolfgang: Wandlungen im humanen Selbstverständnis. In: Schmidinger, Heinrich/Clemens Sedmak (Hg.): Der Mensch - ein ,animal rationale“? Darmstadt 2004, S. 48-70.

Welt ohne Menschen? Transhumanismus: Chance oder Albtraum? Dokumentation von Philippe Borel (1:36:32), Erstausstrahlung: arte 23.10.2012; YouTube http://www. youtube.com/watch? $\mathrm{v}=$ YG0Kynyepzs

Wimmer, Klaus-Michael: Der Andere und die Sprache. Vernunftkritik und Verantwortung, Berlin 1988.

Wimmer, Michael: Die Gabe der Bildung. In: Masschelein, Jan/Michael Wimmer: Alterität Pluralität Gerechtigkeit. Randgänge der Pädagogik, Leuven/Sankt Augustin 1996, S. 127-162.

Zons, Raimar: Die Zeit des Menschen. Zur Kritik des Posthumanismus, Frankfurt am Main 2001, 7-30.

Zymek, Bernd: Was bedeutet „Ökonomisierung der Bildung“? Analyse des Gutachtens der Vereinigung der Bayerischen Wirtschaft „Bildung neu denken! Das Zukunftsprojekt““. In: Berliner Debatte INITIAL 16 (2005) 4, S. 3-13. 